\title{
Auto-diagnosis of COVID-19 using Lung CT Images with Semi-supervised Shallow Learning Network
}

\author{
Debanjan Konar ${ }^{1,2}$, (Member, IEEE), Bijaya K. Panigrahi ${ }^{1}$,(Senior Member, IEEE), \\ Siddhartha Bhattacharyya ${ }^{3}$, (Senior Member, IEEE), Nilanjan Dey ${ }^{4}$, (Senior Member, IEEE), \\ and Richard Jiang ${ }^{5}$, (Senior Member, IEEE) \\ ${ }^{1}$ Department of Electrical Engineering, Indian Institute of Technology Delhi, New Delhi, India, Email: (e-mail: Debanjan.Konar@ee.iitd.ac.in, and \\ bkpanigrahi@iitd.ac.in) \\ ${ }^{2}$ Department of Computer Science and Engineering, Sikkim Manipal Institute of Technology, Sikkim Manipal University, Sikkim, India \\ ${ }^{3}$ Department of Computer Science and Engineering, CHRIST (Deemed to be University), Bangalore, India (e-mail: dr.siddhartha.bhattacharyya @ gmail.com) \\ ${ }^{4}$ Department of Computer Science and Engineering, JIS University, Kolkata, India (e-mail: nilanjan.dey@jisuniversity.ac.in) \\ ${ }^{5}$ School of Computing and Communications, Lancaster University, Lancaster, UK (e-mail: r.jiang2@lancaster.ac.uk) \\ Corresponding author: Siddhartha Bhattacharyya (e-mail: dr.siddhartha.bhattacharyya@gmail.com).
}

This work was partially supported by EPSRC Grant (EP/P009727/1) and Leverhulme Trust Grant (RF-2019-492)

\begin{abstract}
In the current world pandemic situation, the contagious Novel Coronavirus Disease 2019 (COVID-19) has raised a real threat to human lives owing to infection on lung cells and human respiratory systems. It is a daunting task for the researchers to find suitable infection patterns on lung CT images for automated diagnosis of COVID-19. A novel integrated semi-supervised shallow neural network framework comprising a Parallel Quantum-Inspired Self-supervised Network (PQIS-Net) for automatic segmentation of lung CT images followed by Fully Connected (FC) layers, is proposed in this article. The proposed PQISNet model is aimed at providing fully automated segmentation of lung CT slices without incorporating pre-trained convolutional neural network based models. A parallel trinity of layered structure of quantum bits are interconnected using an $\mathcal{N}$-connected second order neighborhood-based topology in the suggested PQIS-Net architecture for segmentation of lung CT slices with wide variations of local intensities. A random patch-based classification on PQIS-Net segmented slices is incorporated at the classification layers of the suggested semi-supervised shallow neural network framework. Intensive experiments have been conducted using three publicly available data sets, one for purely segmentation task and the other two for classification (COVID-19 diagnosis). The experimental outcome on segmentation of CT slices using self-supervised PQIS-Net and the diagnosis efficiency (Accuracy, Precision and AUC) of the integrated semi-supervised shallow framework is found to be promising. The proposed model is also found to be superior than the best state of the art techniques and pre-trained convolutional neural network-based models, specially in COVID-19 and Mycoplasma Pneumonia (MP) screening.
\end{abstract}

INDEX TERMS COVID-19, QIS-Net, Lung CT Image segmentation, 3D-UNet and ResNet50.

\section{INTRODUCTION}

$\mathbf{T}$ he world has suffered a lot in the recent pandemic due to the 2019 novel coronavirus disease (COVID19) since its rapid outbreak from Wuhan, China. There have been sharp rises in infected and suspected cases in almost all the countries in the world from the beginning of January 2020 as reported by World Health Organization [1]. The severe effect of coronavirus disease has inflicted a SARSCoV-2 acute respiratory syndrome and has resulted in a new febrile respiratory tract illness. Despite imposition of various strict measures and physical isolation guidelines, the number of positive test cases is rising rapidly and as of today $(22 / 12 / 2020)$, the total number of confirmed cases reported in the entire world is $76,023,488$ [2]. There are mainly three standard widely used diagnosis procedures viz. Reverse Transcription Polymerase Chain Reaction (RT-PCR) test from swab samples, Chest X-ray and Lung CT scan images for COVID-19 detection [3]. However, the real-time RT-PRT test using detection of nucleotide has reported low sensitivity in China and hence it is not an effective tool for 
coronavirus infection detection [4] owing to lack of stability, quality and viral materials in specimens. In addition, lack of testing capabilities in the underdeveloped countries owing to insufficient test kits has spurred the demand for alternative COVID-19 diagnosis. The potential alternatives to RT-PRT test based COVID-19 detection include methods used on Lung CT scan image and Chest X-ray image segmentation. The captured lung CT scans of COVID-19 infected patients often show a bilateral patchy shadow. Moreover, Chest CT scan is a noninvasive and a fast diagnosis procedure and reported high sensitivity for pre-screening of COVID-19 infections [4]. However, with rise in the number of infections and suspected cases, it is a paramount and laborious task for the health experts to manually annotate the infected lesions and manually contour them in the current worldwide pandemic situation. In these circumstances, in order to maximize the diagnosis of the infected patients and to improve the treatment access, it is always preferred to have an automatic and robust segmentation technique followed by assessment of coronavirus infections.

\section{A. RELATED WORKS}

Recent years have witnessed the progress of deep learning technologies in the field of medical image segmentation which have become popular diagnostic tools due to key feature representation [5]-[10]. In this year, a plethora of deep learning networks have been employed for automatic detection of COVID-19 pneumonia lung CT volumes and have reported promising accuracy [11]-[15]. A multi-objective differential evolution assisted convolutional neural network (CNN) [12] is suggested for COVID-19 lung CT image classification by leveraging the hyper-parameters of the CNN. Zheng et al. [16] proposed a weakly supervised deep learning model with the pre-trained U-Net for COVID-19 infection detection using lung CT volumes and reported high accuracy, sensitivity and specificity. Yan et al. [17] introduced a convolutional neural network introducing Progressive Atrous Spatial Pyramid Pooling to address the sophisticated infected lesions with overlapping and with wide variations of shape and orientation of lung CT volumes. However, owing to lack of sufficient annotated lung CT images and lack of image specific adaptability for unforeseen lung CT image classes (the infections on lung CT images vary with regions), the pre-trained CNN models fail to achieve desired accuracy. In addition to this, requirement of high computational resources to train the aforementioned deeply supervised networks is seldom a cost effective solution for COVID-19 diagnosis. To avoid the over-fitting during training of CNN based models with small data sets, a latent representation learning exploring multiple features prevalent to lung CT volumes, is suggested by Kang et al. [15]. In addition to this, a simple neural network model incorporating two coupled 3D Res-Nets with prior attention learning is proposed by Wang et al. [18]. An attention-based deep 3D multiple instance learning with weak labels of chest CT images is proposed for COVID19 screening [19]. In spite of being relatively less complex models for COVID-19 infected lung CT image segmentation, these approaches rely on extensive feature learning during training.

In this article, we have proposed an integrated semisupervised shallow learning network model comprising a Parallel Quantum-Inspired Self-Supervised Neural Network (PQIS-Net) followed by fully connected classification layers for COVID-19 diagnosis. Of late, the authors have proposed quantum-inspired self-supervised networks referred to as QIS-Net [20] and QIBDS Net [21] for automatic brain lesion segmentation. Authors have also developed the optimized version of QIBDS Net referred to as Opti-QIBDS Net [22] which is found suitable for brain tumor segmentation. These self-supervised network architectures which are tailored and tested on brain MR images and efficient in brain MR image segmentation serve as the inspiration behind the current work. In this manuscript, we aim to further investigate the parallel version of QIS-Net [20] on COVID-19 infected lung CT images without any sort of supervision or training for segmentation followed by classification using fully connected layers for feasibility analysis on COVID-19 diagnosis.

\section{B. CONTRIBUTIONS}

Eventually, in the current pandemic situation in the world, it is an uphill task for the health care professionals to acquire large volumes of lung CT images with annotations for deep supervision. Hence, the primary focus of the paper is to offer a potential alternative to deeply supervised networks using a semi-supervised shallow neural network model composed of a fully parallel self-supervised network (PQIS-Net) for appropriate segmentation for tiny COVID-19 infected lesions and fully connected (FC) layers at the end for enabling training on weak data labels for suitable assessment of COVID19 infections. The significant four-fold contributions of the article are highlighted as follows:

1) The convergence of the QIS-Net architecture is relatively slower than the Fully Connected (FC) layers and hence, there is a imbalance in the processing of segmentation using QIS-Net and classification at FC layers. To obviate the problem, we have proposed a parallel version of QIS-Net architecture referred to as PQIS-Net, which is found suitable for the segmentation of lung CT volumes with COVID-19 infections without any sort of supervision or training. PQIS-Net takes the CT volume (equal to batch size $\mathcal{B}$ ) as inputs under parallel architecture, whereas QIS-Net takes brain MR image slices one at a time.

2) An $\mathcal{N}$-connected neighborhood topology-based segmentation using PQIS-Net for taking into cognizance the wide variations of local intensities of lung CT images is the key contribution of the proposed work.

3) We have also modified the loss or error function incorporated in QIS-Net which is based on the summation of the differences of interconnection weights between two successive iterations. Moreover, the inputs and weights of the PQIS-Net model are represented in terms of 
frequencies (cos and sine components) or spectral components in quantum formalism, thereby enabling faster convergence than QIS-Net which relies fully on quantum weights.

4) In addition, selections of $p$-random $2 \mathrm{D}$ patches from the PQIS-Net segmented images are allowed to augment the limited training data sets with high representation features to be fed to the constituent FC layers for processing (training) thereby obviating over-fitting.

Rigorous experiments have been carried out considering two different publicly available data sets of COVID-19 lung CT images, one purely for segmentation task and the other one for segmentation followed by classification to facilitate an accurate diagnosis. The extensive experimental results validate our proposed semi-supervised shallow neural network model which outperforms the state of the art pre-trained CNN models with weak annotations, thus promoting autodiagnosis with self-supervised neural network models.

The remaining portion of the manuscript is organized as follows: The proposed semi-supervised shallow neural network model comprising PQIS-Net architecture and its operation with the fully connected layers are discussed in Section II. Experimental results and discussions including the data set details, experimental setup are provided in Section III. Finally, the concluding remarks are confabulated in Section IV.

\section{PROPOSED SEMI-SUPERVISED SHALLOW NEURAL NETWORK MODEL}

The Parallel Quantum-Inspired Self-Supervised Neural Network (PQIS-Net) is the core of the proposed semi-supervised shallow neural learning model which is combined with fully connected layers at the end for classification and diagnosis of COVID-19 disease. The PQIS-Net is employed to segment lung CT image slices which are infected by COVID-19 or pneumonia in parallel fashion thereby reducing processing time. The PQIS-Net segmented images form highly representative features for classification. An integrated semisupervised shallow learning model incorporating the selfsupervised PQIS-Net with Fully Connected (FC) layers at the end is targeted to be developed which is appropriate for training at the FC layers with limited training data sets and can offer accurate diagnosis. The classification outcome is obtained using a majority voting scheme. A schematic outline of the proposed integrated self-supervised shallow neural network model with the fully connected layers is illustrated in Fig. 1. The following subsection II-A sheds light on the detailed description of our previously developed quantum-inspired fully self-supervised neural networks [20], [21]. A short description about FC layers is also provided in subsection II-C.

\section{A. PARALLEL QUANTUM-INSPIRED SELF-SUPERVISED NEURAL NETWORK (PQIS-NET) FOR SEGMENTATION}

The Parallel Quantum-Inspired Self-Supervised Neural Network is the extended parallel version of our previous network architectures [20], [21]. Each one of the constituent network architectures in PQIS-Net comprises a trinity of layers of quantum neurons (represented as quantum bits or qubits). The incorporation of quantum-inspired computing in the suggested PQIS-Net stems from the fact that the classical self-supervised networks suffer from convergence problems [23]-[25]. The incorporation of quantum-inspired computing in the suggested parallel quantum-inspired selfsupervised neural networks enables faster convergence by reducing the number of epochs with forceful termination and hence yields better accuracy in segmentation tasks [20]-[22], [26]-[28]. The network dynamics of PQIS-Net replicate the basic operation of the QIS-Net model [20] in parallel. The basis computation unit for the PQIS-Net is a quantum bit or a qubit designated by a quantum neuron in all trinity of layers in the architecture in matrix notation. One such layer matrix comprising qubits is shown out of the identical parallel layers in the PQIS-Net as follows.

$$
\left[\begin{array}{ccccc}
\left|\phi_{11}^{l}\right\rangle & \left|\phi_{12}^{l}\right\rangle & \left|\phi_{13}^{l}\right\rangle & \ldots & \left|\phi_{1 m}^{l}\right\rangle \\
\ldots & \ldots & \ldots & \ldots & \ldots \\
\ldots & \ldots & \ldots & \ldots & \ldots \\
\ldots & \ldots & \ldots & \ldots & \ldots \\
\left|\phi_{n 1}^{l}\right\rangle & \left|\phi_{n 2}^{l}\right\rangle & \left|\phi_{n 3}^{l}\right\rangle & \ldots & \left|\phi_{n m}^{l}\right\rangle
\end{array}\right]
$$

Hence, each qubit is designated as $\phi_{i j}^{l}$ at the $l^{\text {th }}$ layer of the network architecture. The network layers are interconnected through 8-connected spatial neighborhood neuron subsets and serve as the significant characteristic of the network architecture. In each layer of the PQIS-Net architecture, quantum neurons are also intra-linked among themselves with intra-connection strengths $\frac{\pi}{2}$ (quantum 1 logic). The $\mathcal{N}$-connected neighborhood information of each candidate pixel is propagated to the subsequent layers for further computation in forward (input to hidden and hidden to output layer) and in counter-propagation fashions. The counter-propagation obviates the quantum back-propagation procedure thereby enabling faster convergence and reduced time complexity.

The principle of operation of the network is as follows. Each neuron of the network layers is designated as a qubit or a quantum bit and the inter-linked weights and its corresponding activation are mapped using rotation gates operating on the qubits. The classical input image pixels $\left(x_{i}^{l}\right)$ at layer $l$ are converted to quantum bits as

$$
\left|\phi_{i}^{l}\right\rangle=\left[\begin{array}{c}
\cos \left(\frac{\pi}{2} x_{i}^{l}\right) \\
\sin \left(\frac{\pi}{2} x_{i}^{l}\right)
\end{array}\right] i=1, \ldots m \times n
$$

The rotation gates are employed to update the qubit with rotation angle for inter-connection strength and activation as $\omega^{l}$ and $\gamma^{l}$ (say) at layer $l$, respectively. The angle of rotation of an interconnection strength, $\omega_{i, j}^{l}$ is decided by the adaptive and relative difference of fuzzy membership measures between the candidate pixel $(i)$ and its corresponding neighborhood $(j)$ located at its $\mathcal{N}$-connected region in quantum formalism. The inspiration behind the adaptive and relative fuzzy membership measures in the evaluation of the rotation angle is to distinguish between the foreground and 


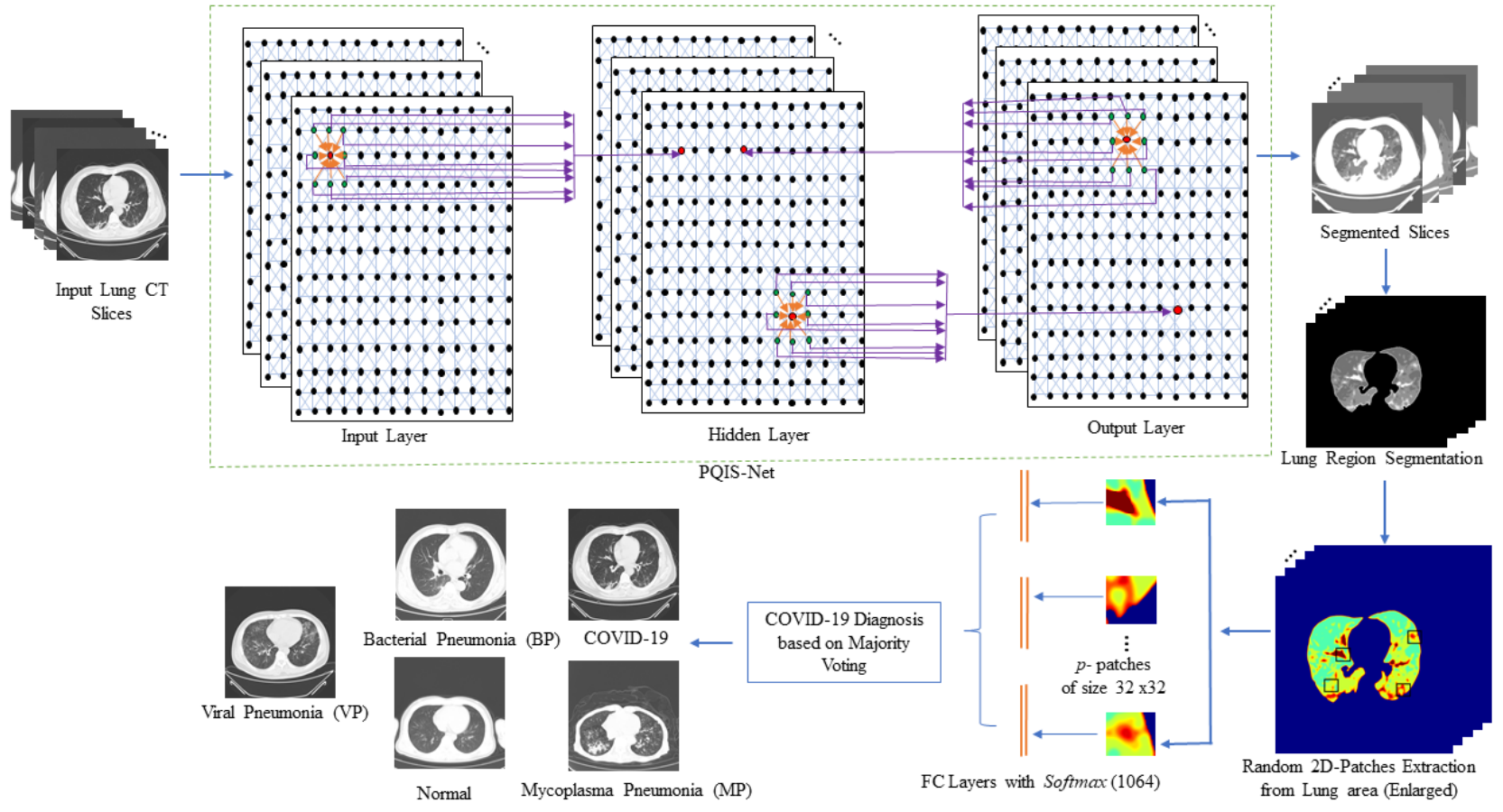

FIGURE 1: A Parallel Quantum-Inspired Self-Supervised Network (PQIS-Net) assisted semi-supervised shallow learning framework for COVID-19 diagnosis (only three inter-layer connections are shown for clarity and gray-scale segmented slices are color mapped for better visibility).

background image pixels. The angle of rotation is evaluated as

$$
\omega_{i, j}^{l}=1-\left(\mu_{i}^{l}-\mu_{i, j}^{l}\right) ; i \in m \times n, j \in\{1,2, \ldots \mathcal{N}\}
$$

Here, the fuzzy graded input at the $i^{\text {th }}$ candidate neuron and its corresponding spatially $\mathcal{N}$-connected second-order neighborhood neuron at layer $l$ are $\mu_{i}^{l}$ and $\mu_{i, j}^{l}$, respectively. It clearly describes the relative difference in the fuzzy membership measures between the foreground $\left(\mu_{i}^{l}\right)$ and the background regions $\left(\mu_{i, j}^{l}\right)$. Eg., if $\mu_{i}^{l}=1$ and $\mu_{i, j}^{l}=0$ then $\omega_{i, j}^{l}=1-\left(\mu_{i}^{l}-\mu_{i, j}^{l}\right)=0$ suggests there is no change in rotation angle (activation remains same) and in this case, it is already segmented. On contrary, if $\mu_{i}^{l}=1$ and $\mu_{i, j}^{l}=1$ then $\omega_{i, j}^{l}=1-\left(\mu_{i}^{l}-\mu_{i, j}^{l}\right)=1$ suggests there is a significant change in rotation angle (activation is high). A single qubit is updated using a rotation gate with an angle $\omega^{l}$ as

$$
\left[\begin{array}{c}
\phi_{0^{\prime}}^{l} \\
\phi_{1^{\prime}}^{l}
\end{array}\right]=\left[\begin{array}{cc}
\cos \left(\frac{\pi}{2} \omega^{l}\right) & -\sin \left(\frac{\pi}{2} \omega^{l}\right) \\
\sin \left(\frac{\pi}{2} \omega^{l}\right) & \cos \left(\frac{\pi}{2} \omega^{l}\right)
\end{array}\right] \times\left[\begin{array}{c}
\phi_{0}^{l} \\
\phi_{1}^{l}
\end{array}\right]
$$

The fuzzy context sensitive activation in quantum formalism enables the bi-directional propagation (forward propagation and counter propagation). It is denoted at a layer $l$ of a candidate neuron (pixel) $i$ by $\xi_{i}^{l}$ as

$$
\left|\xi_{i}^{l}\right\rangle=\left[\begin{array}{c}
\cos \gamma_{i}^{l} \\
\sin \gamma_{i}^{l}
\end{array}\right]
$$

where, the angle of rotation for activation $\xi_{i}^{l}$ is $\gamma_{i}^{l}$ measured as the contribution of its $\mathcal{N}$-connected neighborhood neurons as

$$
\gamma_{i}^{l}=2 \pi \times\left(\sum_{j} \mu_{i, j}^{l}\right)
$$

The network input-output dynamics of a basic quantum neuron $(i)$ in the self-supervised PQIS-Net is defined at the layer $l$ as

$$
\left|y_{i}^{l}\right\rangle=\sigma_{P Q I S-N e t}\left(\sum_{j}^{8} f\left(y_{i}^{l-1}\right)\left\langle\varphi_{j}^{l} \mid \xi_{j}^{l}\right\rangle\right)
$$

i.e.,

$$
\begin{array}{r}
\left|y_{i}^{l}\right\rangle=f\left(\frac{\pi}{2} \delta_{i}^{l-1}-\arg \left\{\sum_{j}^{8} f\left(\omega_{j, i}^{l-1}\right) f\left(y_{i}^{l-1}\right)-f\left(\xi_{i}^{l-1}\right)\right\}\right) \\
=\sigma_{P Q I S-N e t}\left(\sum _ { j } ^ { 8 } f ( y _ { i } ^ { l - 1 } ) \left\{\cos \left(\left(\omega_{j, i}^{l-1}\right)-\gamma_{i}^{l-1}\right)+\right.\right. \\
\left.\left.\tau \sin \left(\left(\omega_{j, i}^{l-1}\right)-\gamma_{i}^{l-1}\right)\right)\right\}
\end{array}
$$

Hence, the output at the $i^{t h}$ quantum neuron is depicted as $y_{i}^{l}$ and the phase transformation parameters are denoted as $\delta_{i}^{l-1}$ 
( $\tau$ is an imaginary number).

$$
\begin{array}{r}
\left|y_{i}^{l}\right\rangle=\sigma_{P Q I S-N e t}\left(\sum_{j}^{8} f\left(\frac{\pi}{2} y_{j}^{l}\right)\left\langle\varphi_{j i}^{l} \mid \xi_{j}^{l}\right\rangle\right) \\
=\sigma_{P Q I S-N e t}\left(\sum _ { j } ^ { 8 } f \left(\frac{\pi}{2} \times \sigma_{P Q I S-N e t}\left(\sum_{l}^{8} f\left(\frac{\pi}{2} y_{j}^{l}\right)\right.\right.\right. \\
\left.\left.\left\langle\varphi_{k j}^{l} \mid \xi_{k}^{l}\right\rangle\right)\left\langle\varphi_{j i}^{l} \mid \xi_{j}^{l}\right\rangle\right)
\end{array}
$$

$\varphi_{j i}^{l}$ and $\varphi_{k j}^{l}$ are the interconnection weights between input to intermediate and intermediate to output layers, respectively.

$$
\begin{array}{r}
\sigma_{P Q I S-N e t}\left(\sum _ { j } ^ { 8 } f \left(\frac{\pi}{2} \times \sigma_{P Q I S-N e t}\left(\sum_{k}^{8} f\left(\frac{\pi}{2} y_{j}^{l}\right) \cos \left(\omega_{k j}^{l}-\gamma_{k}^{l}\right)\right.\right.\right. \\
\left.\left.\left.\cos \left(\omega_{j i}^{l}-\gamma_{j}^{l}\right)+\tau \sin \left(\omega_{k j}^{l}-\gamma_{k}^{l}\right) \sin \left(\omega_{j i}^{l}-\gamma_{j}^{l}\right)\right)\right)\right)
\end{array}
$$

The $\sigma_{P Q I S-N e t}$ activation function [Quantum Multi-level Sigmoidal (QMSig)] employed in the above Equation is defend as [20], [21]

$$
\sigma_{P Q I S-N e t}\left(z ; \lambda_{\theta}, \mathcal{S}_{\theta}, \gamma\right)=\sum_{\theta=1}^{L} \frac{1}{\lambda_{\theta}+e^{-\nu\left(z-(\theta-1) \mathcal{S}_{\theta-1}-\gamma\right)}}
$$

where,

$$
\lambda_{\theta}=\frac{\mathcal{N}_{s}}{\mathcal{S}_{\theta}-\mathcal{S}_{\theta-1}}
$$

Hence, the outcome of two adjacent classes viz., $\theta$ and $\theta-1$ are $\mathcal{S}_{\theta}$ and $\mathcal{S}_{\theta-1}$, respectively and the sum of the contribution of the $\mathcal{N}$-connected neighborhood pixels is designated as $\mathcal{N}_{s}$. $\nu$ denotes the steepness factor of the function and $L$ is the number of gray levels in the segmented image.

A coherent network error cum loss function is introduced in PQIS-Net and is evaluated as [20] where, $\varphi\left(\omega_{i, j}^{\iota, k}, \gamma_{i}^{\iota, k}\right)$ is the weighted inter-connection of $k^{t h}$ parallel layer at a particular epoch $\iota$, which is linearly dependent on $\omega$ and $\gamma$. $\mathcal{B}$ is the batch size of the constituent PQIS-Net and the semisupervised model. The convergence analysis of the proposed PQIS-Net is demonstrated in the Appendix section.

\section{B. OPTIMIZATION PROCEDURE OF PQIS-NET}

The activation parameter $\gamma$ used in the QMSig activation function $(\sigma)$ is appropriate for uniformly distribution of intensity and hence, gray-level segmentation accuracy degrades for lung CT slices due to wide variations of gray-scales having heterogeneous response exhibited over the $\mathcal{N}$-connected region. In order to tackle this problem, we have entrusted on adaptive and optimal thresholding schemes using Quantuminspired Differential Evolution [29] with Otsu's [30] multilevel thresholding as fitness function. There are four distinct adaptive activation schemes used for the activation parameter $\gamma$ in the proposed QMSig activation function as provided below [20], [25].

(1) Activation based on $\beta$-distributed intensity of $\mathcal{N}$ connected neighborhood image pixels $\left(\gamma_{\beta}\right)$.
(2) Activation based on skewness $\left(\gamma_{\chi}\right)$.

(3) Activation based on fuzzy graded pixel heterogeneous intensity of 8-connected neighborhood $\left(\gamma_{\xi}\right)$.

(4) Activation based on fuzzy cardinality estimation of 8connected neighborhood $\left(\gamma_{\nu}\right)$.

The optimized multi-class level, $\left(\mathcal{L}_{\theta}\right)$ for fixed number of boundaries or class $\mathcal{L}$ is defined in a closed set $\mathcal{F}_{\lambda_{\omega}}$ as [20]

$$
\mathcal{F}_{\lambda_{\theta} \mathcal{L}}=\left\{\left\{\lambda_{\theta \mathcal{L}}\right\}, \mathcal{L}=4,5,6,7,8\right\}
$$

In order to obtain a number of optimal thresholds $\left\{\theta_{1}, \theta_{2}, \cdots, \theta_{C_{l}-1}\right\}$, Otsu's multi-level image threshold$=$ ing [30] is incorporated to maximizes the spread of the classes, and is defined as [30]

$$
\mathcal{O}=f_{n}\left\{\theta_{1}, \theta_{2}, \cdots, \theta_{C_{l}-1}\right\}=\sum_{k=1}^{C_{l}} w_{k}\left(r_{k}-r\right)
$$

where, $C_{l}$ represents the number of defined classes in $\mathcal{C}$ $=\left\{C_{1}, C_{2}, \ldots, C_{C_{l}}\right\}$ and

$$
w_{k}=\sum_{i \in C_{k}} p_{i}, r_{k}=\sum_{i \in C_{k}} i p_{i} / w_{k}
$$

where, $p_{i}$ designates the the $i^{\text {th }}$ pixel and $w_{k}$ represents the probability of class $C_{k}$ with the mean value given by $\mu_{k}$. The mean of the class $\mathcal{C}$ is given by $m$. In this work, for each multi-class level $\mathcal{L}=\{4,6,8\}$ four sets $\mathcal{F}_{\lambda_{\theta \mathcal{L}}}=\left\{S_{1}, S_{2}, S_{3}, S_{4}\right\}$ of class boundary are computed using Otsu's method [30] as fitness values in quantum formalism and are optimized using quantum-inspired differential evolution (QDE).

\section{FULLY-CONNECTED (FC) LAYERS FOR PATCH-BASED CLASSIFICATION}

The segmented lung CT images by PQIS-Net are targeted for classification using Fully Connected (FC) layers to enable the diagnosis of COVID-19 or pneumonia (Non-Covid). However, to avoid over-fitting in the FC layers due to large size of segmented image features, patch-based classification [31], [32] is preferred incorporating $p$-number of random patches with relatively small fixed size window of $s \times s$. The patches extracted from the segmented lung CT images are augmented with the limited training data and hence avoids overfitting. In addition, patch-based training at the FC layers relatively reduces the network complexity. The center pixel $x_{p}$ of an image patch $\mathcal{R}_{p}$ is randomly chosen from the segmented lung region (the lung and the background pixels significantly differ in their intensities) to obviate the empty region of the segmented image without using any lung mask. The value of $p$ is chosen judiciously such that each pixel of the segmented lung CT image is considered at least once. Each patch as a vector of $s^{2}$ pixels is concatenated along a fixed lexicographic ordering [33]. It may be noted that each image pixel from the infected lung region of the $p$-random patches may be considered as the highest representative feature for classification. Some patches contain white and black pixels without any significant feature (infection region) 
or small section of an infected lesion for classification. In what follows, each image patch as vector is fed to the FC layers for classification with a Softmax function and out of $p$-outcomes the final decision is made by a majority voting scheme [34]. For simplicity, in the experimental setup, maximum of 100 patches for classification are allowed with patch size of $32 \times 32$. Here, we have employed cross entropy loss for COVID-19 diagnosis. The loss $\left(\mathcal{L}_{(\Theta)}\right)$ is computed by leveraging the hyper-parameters $\Theta$ of the semi-supervised neural network model. It is defined as

$$
\left.\left.\underset{\Theta}{\operatorname{argmin}} \mathcal{L}_{(\Theta)}=\sum_{i}^{\mathcal{C}}\left[t_{i} \log y_{(} \alpha_{i}\right)+\left(1-t_{i}\right) \log \left\{1-y_{(} \alpha_{i}\right)\right\}\right]
$$

where, $y\left(\alpha_{i}\right)$ is the predicted outcome of the FC layers on input $\alpha_{i}$ with respect to the network hyper-parameter set $\Theta$. $t_{i}$ is the target output.

\section{RESULTS AND DISCUSSIONS}

\section{A. DATA SET}

Publicly available lung CT images are collected from three data sources [35]-[37] and experiments are performed using the proposed semi-supervised neural network model on both the data sets. One of the data sets [35] contains total 2482 lung CT images with variable sizes and out of these, 1252 lung CT images are infected by COVID-19 and 1230 CT slices are not infected by COVID-19. It may be noted that non-COVID CT slices includes few healthy slices which lack significant distinguishable features for diagnosis. We have augmented the COVID-19 infected and non-COVID lung slices using rotation, scaling, shearing, and flipping operations. The training-validation and testing splits of the randomly chosen images are provided in Table 1 . The Brazilian data set [35] is acquired from the real patients of Sao Paulo, City hospitals, Brazil. Few samples from the Brazil data set [35] are shown in Fig. 2 (COVID-19 infected) and in Fig. 3 (non-COVID infected). Another data set [36] comprises only 20 labelled COVID-19 lung CT volumes of fixed size of $512 \times 512$ which includes infection masks, lung masks (left and right) and lung-infection pair masks. These labelled CT volumes are manually segmented and verified by radiologist experts. The data set [36] comprising the labelled CT volume masks is used in the experiment for the segmentation task. On the contrary, the other data sets [35], [37] are used for segmentation followed by classification tasks. The third data set employed in this experiment is the IEEE CCAP data set [37] collected from IEEE Data port which comprises five different sets of lung CT images (COVID-19, Viral Pneumonia (VP), Bacterial Pneumonia (BP), Mycoplasma Pneumonia (MP) and Normal lung). Randomly selected input CT slices from IEEE CCAP data set [37] are shown in Fig. 4. Details of the data sets used for diagnosis are provided in Table 1.
TABLE 1: Details of the data sets (Training-Validation and Test Data Set) used for classification (number of Patients)

\begin{tabular}{|c|c|c|}
\hline Disease & $\begin{array}{l}\text { Training and } \\
\text { Validation }\end{array}$ & Test Data Set \\
\hline \multicolumn{3}{|c|}{ Brazilian Data Set [35] } \\
\hline$C O V I D-19$ & 868 & 384 \\
\hline non $-C O V I D$ & 896 & 334 \\
\hline \multicolumn{3}{|c|}{ CCAP Data Set [37] } \\
\hline$C O V I D-19$ & $1286(28)$ & $520(14)$ \\
\hline$V P$ & $528(9)$ & $232(4)$ \\
\hline$B P$ & $1172(24)$ & $427(8)$ \\
\hline$M P$ & $544(14)$ & $240(4)$ \\
\hline Normal & $1450(38)$ & $644(12)$ \\
\hline
\end{tabular}

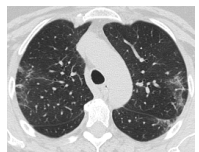

(a) $\# 1$

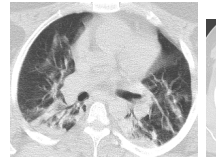

(e) \#242

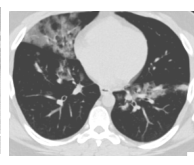

(b) \#27

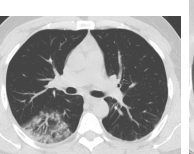

(f) \#343

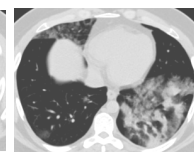

(c) \#35

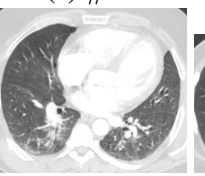

(g) \#434

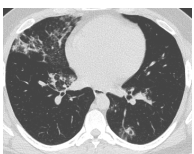

(d) \#55

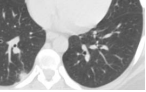

(h) \#1212
FIGURE 2: Randomly selected COVID-19 infected input lung CT slices [35].

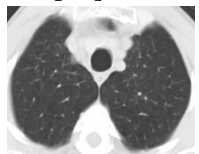

(a) \#3

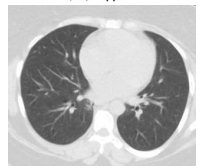

(e) \#290

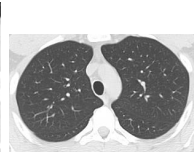

(b) \#28

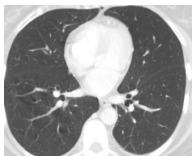

(f) \#317

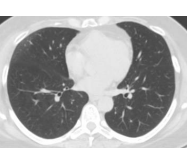

(c) \#121

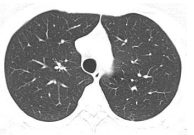

(g) \#842

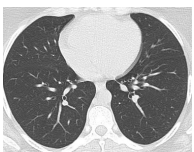

(d) \#186

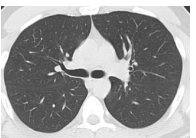

(h) \#1217
FIGURE 3: Randomly selected Pneumonia (Non-COVID) infected input lung CT slices [35].

\section{B. EXPERIMENTAL SETUP}

In this current work, extensive experiments have been carried out on lung CT images of variable sizes using a high performance DL GPU (Nvidia RTX2070) System with MATLAB 2020a and Python 3.6.2 (Pytorch). However, the proposed semi-supervised shallow network framework is implemented without using any sort of GPU support. The Brazilian data set [35] and the IEEE CCAP data set [37] are divided into 7 : 3 ratio for training, validation and testing, respectively for segmentation followed by classification. In addition, experiments are carried out using 5-fold cross validation. The results for these three different scenario (data sets) are investigated by leveraging the set of hyper-parameters of the proposed semi-supervised shallow neural network.

The parallel quantum-inspired self-supervised network (PQIS-Net) is experimented with the pre-processed normal- 


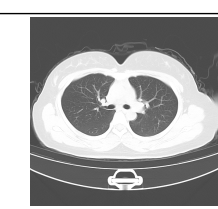

(a) \#4(1)

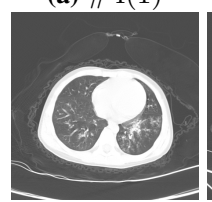

(e) \#5(1)
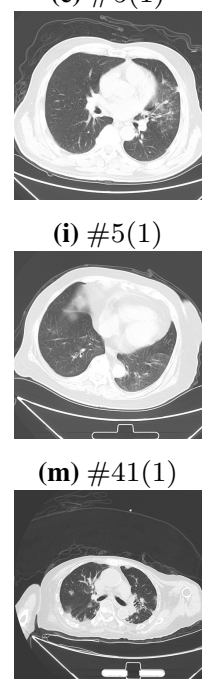

(q) \#24(47) (b) \#6(220)

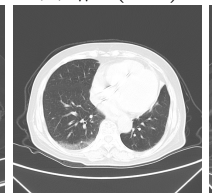

(f) $\# 3(7)$

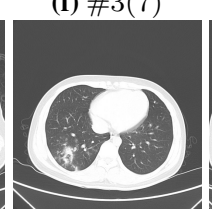

(j) \#12(10)

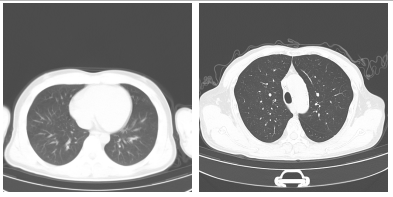

(c) \#18(240)

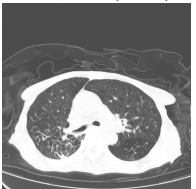

(g) \#40(76)

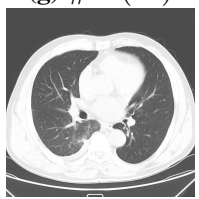

(k) \#46(60)

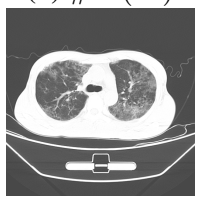

(n) \#58(20)

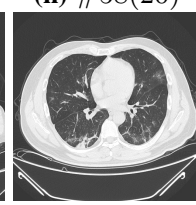

(r) \#25(147) (o) \#22(45)

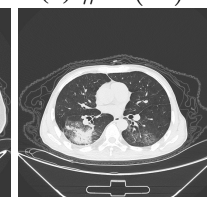

(s) \#24(47)

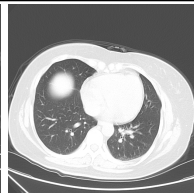

(d) \#24(245)

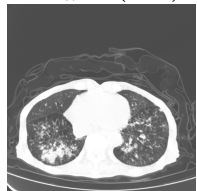

(h) \#90(76)

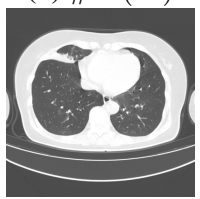

(l) \#51(80)

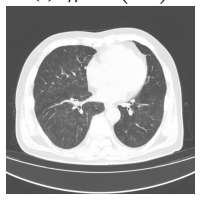

(p) \#81(55)

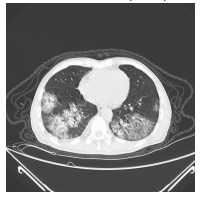

(t) $\# 24(47)$

FIGURE 4: Randomly selected input $(a-d)$ Normal, $(e-h)$ MP, $(i-l) \mathrm{BP},(m-p) \mathrm{VP}$, and $(q-t)$ COVID-19 CCAP lung CT slices (Patent\#) [37].

ized gray-level CT scan images. Pre-processing of the input lung CT images from all three data sets [35]-[37] are performed using normalization of images. The PQIS-Net segmented CT volumes are processed though the 2D binary masks [20], [38] available in the labelled CT volumes in the data set [36] to obtain the infected lesion on lung CT scans in the suggested semi-supervised model. Lung masks are used to segment only lung region from the segmented CT slices, whereas infection masks are considered in the evaluation. The predicted label of each pixel is evaluated based on infection region labelled in the infection masks. Each PQIS-Net segmented image is binarized using Otsu's bi-level thresholding [39] and compared with the binary infection masks. The segmented output images resemble in size with the dimensions of the binary mask and the outcome 1 is considered as infected region and 0 as background (lung region) in diagnosis. Pixel by pixel comparison with the manually segmented regions of interest or lesion mask allows evaluating the dice similarity (DS) which is considered as a standard evaluation procedure in automatic medical image segmentation. The evaluation process using the data set [36] involves the manually segmented lesion (infection region)

mask as ground truth and each 2D pixel is predicted as either True Positive $\left(T_{R P}\right)$ or True Negative $\left(T_{R N}\right)$ or False Positive $\left(T_{R N}\right)$ or False Negative $\left(F_{L N}\right)$. The PQIS-Net is experimented with gray-level CT scan images using with distinct classes $L=4,5,6,7$ and 8 in optimized fashion [22]. In this experiment, the steepness $(\nu)$ in the $\sigma_{P Q I S-N e t}$ activation function, is varied in the range 0.230 to 0.240 with a step size 0.001 . It has been observed that in majority cases, $\nu=0.239$ yields the optimal segmentation. In addition to this, the hyper-parameter $\mathcal{N}$ is chosen intuitively in the current experimental setup and it has been seen that the $\mathcal{N}=8$-connected second order neighborhood pattern yields optimal segmentation using PQIS-Net. It captures the local intensity variations over 8-connected neighborhood regions. In addition to this segmentation, experiments are also set up for classification using the proposed Semi-supervised shallow network model, ResNet50 [9], and 3D-UNet [10] model by replacing the last layer of 3D-UNet [10] and ResNet50 [9] architectures with two fully connected layers. The FC layers in the proposed semi-supervised shallow neural network model are rigorously trained using the stochastic gradient descent algorithm with momentum (SGDM) with an initial learning rate of 0.01 , momentum of 0.8 , minimum batch size of 8 and weight decay of 0.0001 allowing maximum 10 epochs. The convergence of accuracy and loss during training using the proposed semi-supervised shallow neural network with 5-fold cross validation is shown in Fig. 5. The convergence graphs of the suggested self-supervised PQIS-Net is shown in the Appendix along with the convergence graphs of semi-supervised shallow framework during training and validation. Moreover, experiments have been performed on two recently developed CNN architectures suitable for medical image segmentation viz., convolutional 3D-UNet [10] and Residual U-Net (ResNet50) [9] available in GitHub. The ResNet50 [9] and 3D-UNet [10] are rigorously trained using the adam optimizer with an initial learning rate of 0.01 , gradient decay factor of 0.9 and a minimum batch size of 8 allowing maximum 50 epochs to converge. The convergence of accuracy and loss during training using ResNet50 [9] with 5 -fold cross validation is also demonstrated in Fig. 6. The other state of the art techniques include Kang et al. [15], Wang et al. [18], and Han et al. [19] for COVID-19 detection on the Brazilian data set [35] and IEEE CCAP data set [37]. It may be noted that the PQIS-Net segmented slices from IEEE CCAP data set [37] are further prepossessed before classification to remove the cavity regions from the slices in order to obtain lung region with uniform background. In Kang et al. [15], a latent representation-based diagnosis pipeline relying on various heterogeneous features is proposed. In this work, all CT images from the Brazilian data set [35] are pre-processed using a pre-trained V-Net model [40] to segment lung, pulmonary, lung lobes, and infected lesion [41] for feature extraction. To effectively exploit the features, the latent representation learning is implemented using CPM-Nets [42] followed by the latent representation regressor model and classifier for diagnosis of 
COVID-19 and non-COVID CT slices. The suggested framework in Wang et al. [18] involves pre-processing using a pretrained 3D-UNet [10] for segmentation followed by classification using two 3D-ResNets with prior-attention residual learning (PARL). These two 3D-ResNets are employed for pneumonia detection and pneumonia type classification. The pneumonia type classification 3D-ResNet is implemented for binary classification of COVID-19 and non-COVID classes for the Brazilian data set [35]. However, the IEEE CCAP data set [37] comprises various kinds of pneumonia. In order to enable the multi-class diagnosis of pneumonia type classification, these two 3D-ResNets are fused together at the fully connected layers. In Han et al. [19], an attention-based 3D multiple instances learning weakly supervised model is proposed for COVID-19 diagnosis from lung CT images. The suggested model is capable to generate deep 3D instances with semantic representation from raw non-separated bags targeting the infection regions. An attention-based pooling technique combines the $3 \mathrm{D}$ instances into the representation of the bag. Finally, a neural network learning of Bernoulli distributions of the bag levels is transformed into final prediction.

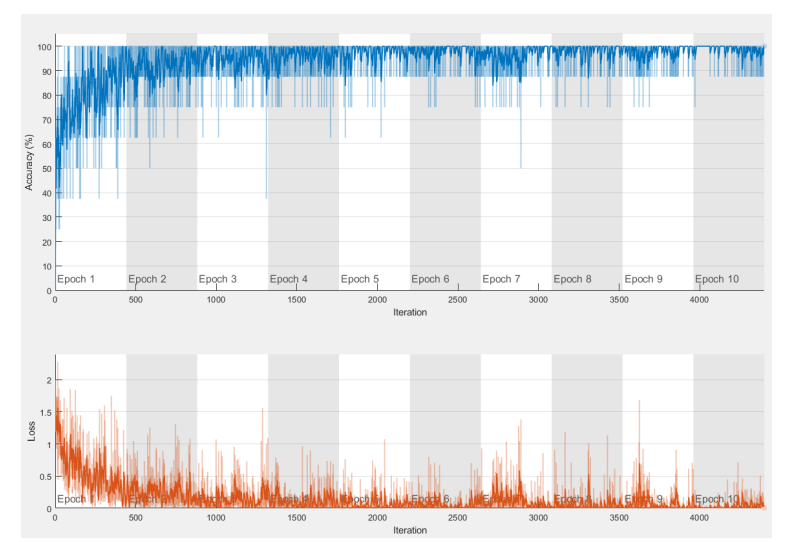

FIGURE 5: Convergence of the proposed semi-supervised shallow neural learning model allowing maximum 10 epochs during training with an initial learning rate $=0.01$ using the IEEE CCAP data set [37].

\section{EXPERIMENTAL RESULTS}

Extensive experiments have been performed in the current setup and experimental outcomes are reported with the demonstration of numerical and statistical analysis on three different data sets [35]-[37]. Segmentation using the proposed semi-supervised shallow neural network, pre-trained ResNet50 [9] and 3D-UNet [10] models have been performed using all the three data sets and segmentation performance is measured on data set [36] using evaluation metrics (ACC, DS, PPV, SS) [43]. The human expert (radiologist) segmented lung and infection masks lung CT image slices of size $512 \times 512$ are provided in Fig. 7 with the input and the PQIS-Net segmented slice. The PQIS-Net is also tested on the Brazilian data set [35] and the IEEE CCAP data

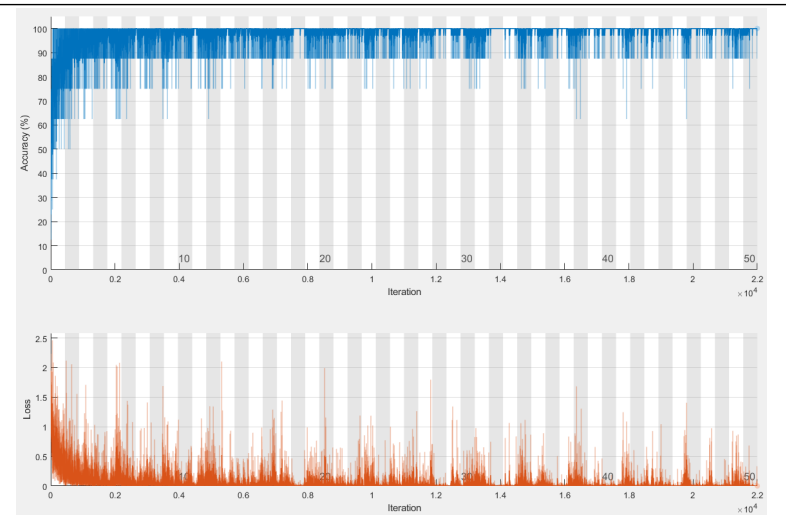

FIGURE 6: Convergence of the ResNet50 [9] model allowing maximum 50 epochs during training with an initial learning rate $=0.01$ and minimum batch size $=8$

set [37]. Segmentation is performed on lung CT slices with two different classes (COVID-19 infected and non-COVID infected) from the Brazilian data set [35] as shown in Fig. 8 and Fig. 9, respectively. In addition, the segmented slices from the IEEE CCAP data set [37] with five distinct classes are also provided in Fig 10. Table 2 reports the segmentation results of the proposed PQIS-Net with ResNet50 [9] and 3DUNet [10] models for three different tasks (infection, lung, infection and lung). It is evident from the experimental data provided in Table 2 and from the statistical significance test (KS test) [20] conducted on the results that in spite of being a self-supervised network and relatively less complex model (in terms of computational resources required to implement) unlike ResNet50 [9] and 3D-UNet [10], the proposed PQISNet attains similar performance in segmentation tasks on the data set [36] in comparison to the pre-trained CNN models (ResNet50 [9] and 3D-UNet [10]) under the four evaluation parameters $(A C C, D S, P P V, S S)$. Table 3 presents the numerical results obtained using the proposed semi-supervised shallow neural network model, Han et al. [19], ResNet50 [9], 3D-UNet [10], Wang et al. [18] and Kang et al. [15] for COVID-19 detection on the Brazilian data set [35]. In addition, experimental results obtained using the IEEE CCAP data set [37] with the proposed semi-supervised shallow network, Han et al. [19], 3D-UNet [10], ResNet50 [9] and Wang et al. [18] are shown in Table 4. The standard evaluation metrics used in Table 3 and Table 4 to measure the COVID-19 detection efficiency are accuracy, precision, recall, F1-score and AUC (Area under ROC curve) [43]. From Table 3, it has been observed that after rigorous tuning of the hyper-parameters, the proposed semi-supervised shallow neural network model outperforms Kang et al. [15] and Wang et al. [18] concerning evaluation metrics used in COVID-19 diagnosis on the Brazilian data set [35]. However, the proposed semi-supervised model has reported similar accuracy as ResNet50 [9] and and Han et al. [19], and precision as 3DUNet [10] while outperforming in terms of other evaluation metrics concerning the outcome of statistical significance 
5

6

7

8

9

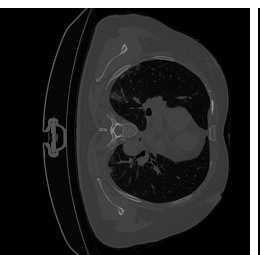

(a) Input

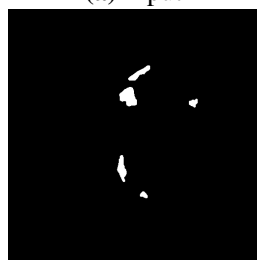

(d) Infection mask

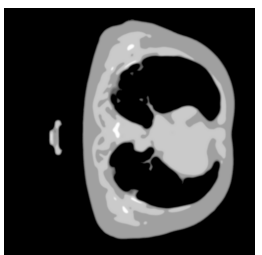

(b) Segmented

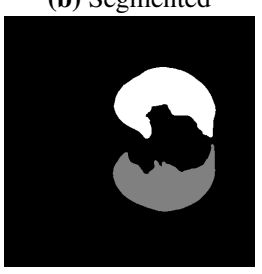

(e) Lung mask

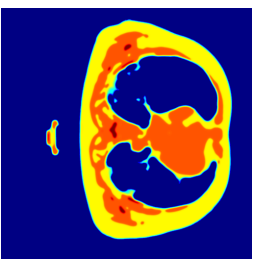

(c) Color-mapping

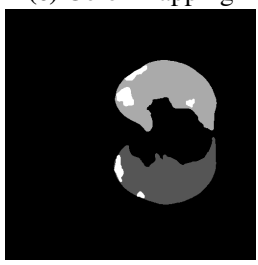

(f) Infection \& Lung mask
FIGURE 7: PQIS-Net segmented lung CT slice\#171 [36] with the three different masks.

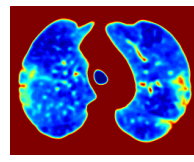

(a) \#1

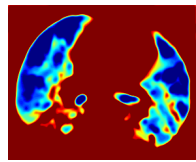

(e) \#242

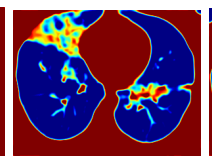

(b) $\# 27$

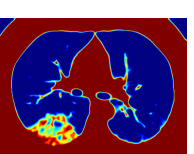

(f) \#343

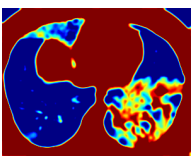

(c) \#35

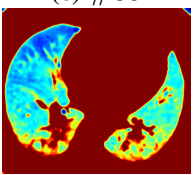

(g) \#434

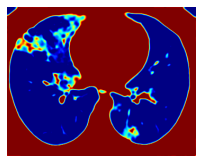

(d) $\# 55$

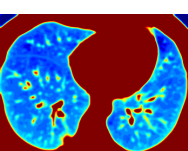

(h) \#1212
FIGURE 8: $(a-h)$ PQIS-Net segmented lung CT slices \# [35] with COVID-19 infections followed by color mapping for class level $L=8$ with activation $\xi$.

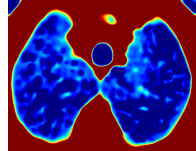

(a) \#3

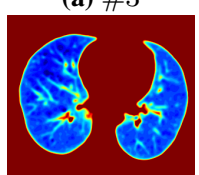

(e) \#290

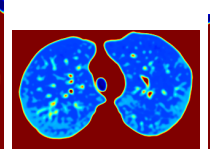

(b) \#28

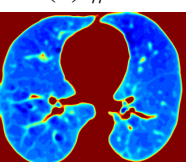

(f) \#317

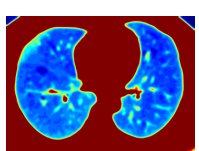

(c) \#121

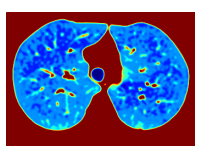

(g) \#842

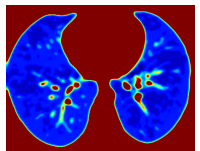

(d) \#186

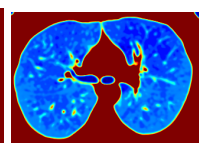

(h) \#1217
FIGURE 9: $(a-h)$ PQIS-Net segmented lung CT slices \# [35] with Pneumonia (Non-COVID) infection followed by color mapping for class level $L=8$ with activation $\xi$.
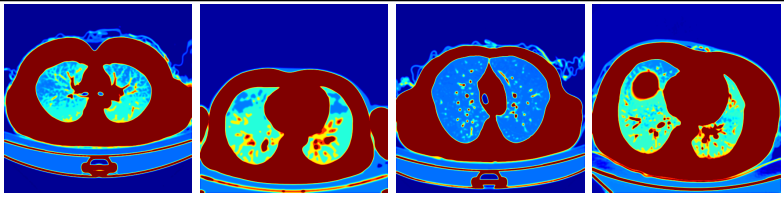

(a) \#4(1)

(b) \#6(220)

(c) \#18(240)

(d) \#24(245)
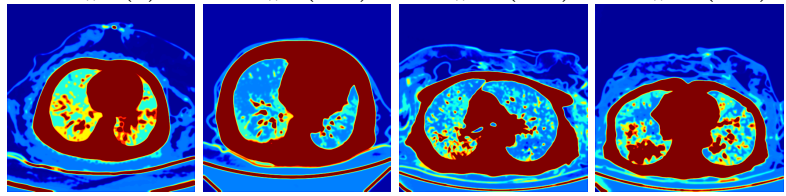

(e) \#5(1)

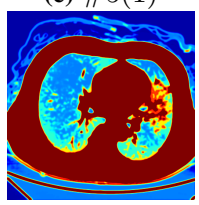

(f) \#3(7)

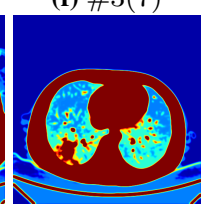

(g) \#40(76)

(h) \#90(76)
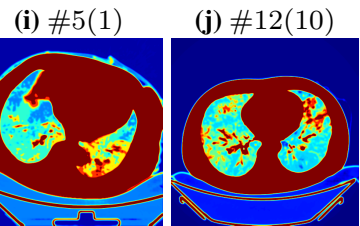

(k) \#46(60)
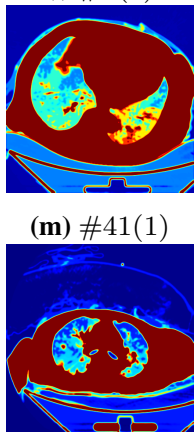

(q) \#24(47)

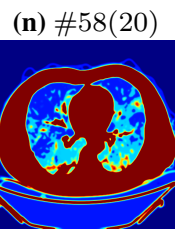

(r) \#25(147)

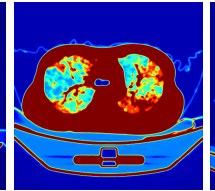

(o) \#22(45)

(p) \#81(55)

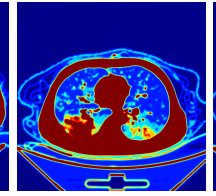

(s) \#24(47)

(t) \#24(47)

FIGURE 10: PQIS-Net segmented followed by color mapping ( $a-$ $d)$ Normal, $(e-h) \mathrm{MP},(i-l) \mathrm{BP},(m-p) \mathrm{VP}$, and $(q-t)$ COVID-19 IEEE CCAP lung CT slices (Patent \#) [37].

KS-test. It is noteworthy that the proposed semi-supervised model using PQIS-Net outperforms 3D-UNet [10] in terms of accuracy, recall, F1-score and AUC in COVID-19 diagnosis on the Brazilian data set [35]. Similarly, the results reported in Table 4 show that the proposed model outperforms Wang et al. [18] and 3D-UNet [10] in terms of the evaluation metrics and reports similar accuracy as ResNet50 [9] and Han et al. in COVID-19 diagnosis on the IEEE CCAP data set [37]. In addition to this, the suggested shallow framework also outperforms Han et al. [19] and ResNet50 [9] in terms of precision and AUC. It is interesting to note from the results reported in Table 3 and Table 4 that with an increase in training samples in data set [37] the improvement of accuracy is phenomenal for all the methods specially 3DUNet [10] and ResNet50 [9]. Thus, despite being a semisupervised shallow learning framework, the suggested semisupervised model has attained better stability on the outcome as is evident from the higher values of precision and AUC while compared with the state of the art best methods in COVID-19 screening.

In addition, the ROC curves and Confusion matrices are reported for quantitative representations of accuracy in 
COVID-19 detection using the Brazilian data set [35] and the IEEE CCAP data set [37] as shown in Fig. 11, Fig. 12, and Fig. 13, and Fig. 14, respectively. The confusion matrices for ResNet50 [9], 3D-UNet [10], Han et al. [19], and Wang et al. [18] in COVID-19 diagnosis on the IEEE CCAP data set [37] are also provided in the Supplementary Materials. Each row and column represents the predicted output class and ground truth target class, respectively. For a good classifier, higher the number of predicted correct samples, the larger will be the values in the diagonal of the confusion matrix. Considering the confusion matrices, it is evident that the suggested shallow framework is superior to the models ResNet50 [9], 3D-UNet [10], Han et al. [19], and Wang et al. [18] in classifying COVID-19 and Mycoplasma Pneumonia (MP) categories. The suggested semi-supervised shallow framework predicts 520 and 237 correctly out of 520 COVID-19 and 240 MP target samples, respectively, whereas ResNet50 [9] has predicted 517 and 235, 3D-UNet [10] has predicted 518 and 235, Han et al. [19] has predicted 519 and 236, and Wang et al. [18] predicted 499 and 231. It further demonstrates that the random patch-based classification incorporated in the suggested semi-supervised framework significantly enhances the performance of COVID-19 screening as the severely infected features present in the COVID-19 slices are captured by these randomly chosen patches from the lung regions. However, the proposed semi-supervised model has maximum miss-classification in Viral Pneumonia (VP) class $(4.8 \%)$ due to the fact that the majority of BP slices looks like Normal CT slices. Despite the remarkable success achieved in COVID-19 screening, ResNet50 [9] and 3DUNet [10] architectures still suffer from some inherent challenges owing to deeper and complex network architectures. ResNet50 [9] and 3D-UNet [10] face slow convergence problems for COVID-19 diagnosis tasks during training. In addition, higher computational (GPU) and memory resources required for ResNet50 [9] and 3D-UNet [10] architectures pose a potential concern in COVID-19 automated diagnosis. On contrary, the proposed semi-supervised model is implemented without any support of Graphics Processing Unit and its convergence is also stabilized at epoch 10 as shown in Fig. 5 and Fig. 2 in the Supplementary Materials, whereas ResNet50 [9] requires 50 epochs to converge as shown in Fig. 6. Hence, it can be concluded, that the performance of the semis-supervised model on lung CT images is statistically significant and offers a potential alternative to the solution of deep learning networks and other time-intensive feature based learning paradigms in future.

\section{CONCLUSION}

In this work, a novel attempt has been made using an integrated semi-supervised shallow neural network encompassing the parallel self-supervised neural network model (PQIS-Net) for fully automatic segmentation of lung CT images followed by fully connected (FL) layers for patchbased classification with majority voting. The PQIS-Net model incorporates the frequency components of the weights

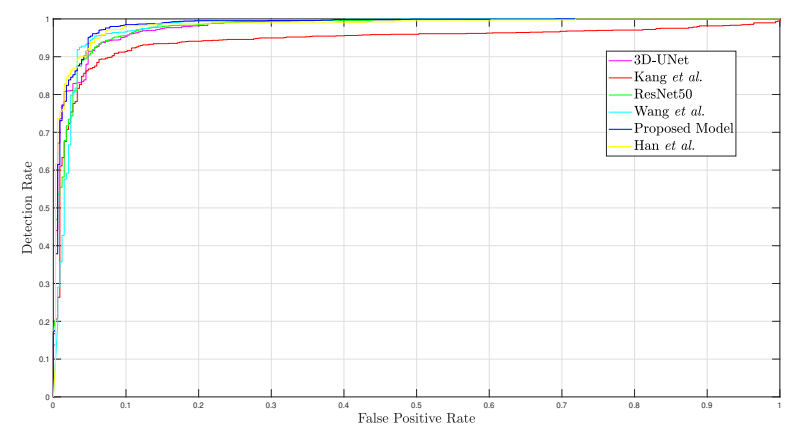

FIGURE 11: ROC Curves for the COVID-19 detection rate vs. false positives using the the proposed semi-supervised shallow neural network model, 3D-UNet [10], ResNet50 [9], Han et al. [19], Kang et al. [15], and Wang et al. [18] on Brazilian data set [35]

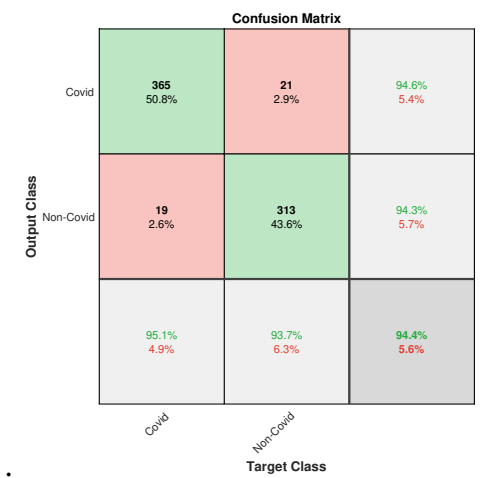

FIGURE 12: Confusion matrix for the accuracy of prediction of COVID-19 using the proposed semi-supervised shallow neural network model on Brazilian data set [35]

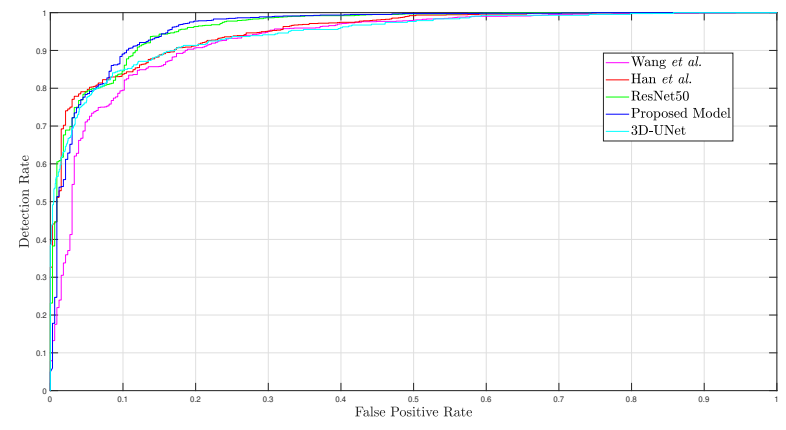

FIGURE 13: ROC Curves for detection rate (in case of of Bacterial Pneumonia (BP), COVID-19, Mycoplasma Pneumonia (MP), Normal lung, and Viral Pneumonia (VP)) vs false positive using the proposed semi-supervised shallow neural network model, ResNet50 [9], 3D-UNet [10], Kang et al. [19], and Wang et al. [18] on IEEE CCAP data set [37]. 
TABLE 2: Average Segmentation Accuracy: $A C C=\frac{T_{R P}+T_{R N}}{T_{R P}+F_{L P}+T_{R N}+F_{L N}}$, Dice Similarity: $D S=\frac{2 T_{R P}}{2 T_{R P}+F_{L P}+F_{L N}}, P P V=$ $\frac{T_{R P}}{T_{R P}+F_{L P}}$ and Sensitivity: $S S=\frac{T_{R P}}{T_{R P}+F_{L N}}$ for the data set [36] using PQIS-Net [One sided non-parametric two sample KS test [44] with $\alpha=0.05$ significance level has been conducted and marked underlined.]

\begin{tabular}{|c|c|c|c|c|c|c|c|c|c|c|c|c|}
\hline \multirow[b]{2}{*}{ Task } & \multirow{2}{*}{\multicolumn{3}{|c|}{ PQIS-Net }} & & \multicolumn{4}{|c|}{ 3D-UNet [10] } & \multicolumn{4}{|c|}{ ResNet50 [9] } \\
\hline & & & & $S S$ & $A C C$ & $D S$ & $P P V$ & $S S$ & $A C C$ & & $P P V$ & $S S$ \\
\hline Lung & 0.989 & 0.841 & 0.793 & 0.972 & 0.990 & 0.871 & $\underline{0.799}$ & 0.918 & 0.989 & 0.853 & 0.751 & 0.940 \\
\hline Infection & $\overline{0.976}$ & 0.790 & $\overline{0.741}$ & 0.920 & 0.987 & $\overline{0.816}$ & $\overline{0.734}$ & 0.899 & $\underline{\underline{0.989}}$ & $\underline{0.833}$ & 0.774 & 0.910 \\
\hline Lung and Infection & 0.988 & 0.811 & 0.773 & 0.885 & 0.990 & $\underline{0.852}$ & 0.767 & 0.968 & 0.989 & $\overline{0.819}$ & 0.794 & 0.946 \\
\hline
\end{tabular}

TABLE 3: Performance analysis of the proposed semi-supervised model, ResNet50 [9], 3D-UNet [10], Han et al. [19], Wang et al. [18] and Kang et al. [15] for COVID-19 detection on the Brazilian data set [35] [One sided non-parametric two sample KS test [44] with $\alpha=0.05$ significance level has been conducted and marked underlined.]

\begin{tabular}{llllll}
\hline Model & Accuracy & Precision & Recall & F1- score & AUC \\
\hline Kang et al. [15] & $0.905 \pm 0.028$ & $0.920 \pm 0.019$ & $0.901 \pm 0.017$ & $0.910 \pm 0.041$ & $0.978 \pm 0.065$ \\
\hline Wang et al. [18] & $0.919 \pm 0.015$ & $0.963 \pm 0.003$ & $0.882 \pm 0.138$ & $0.921 \pm 0.146$ & $0.980 \pm 0.028$ \\
\hline Han et al. [19] & $\underline{0.945} \pm \underline{0.043}$ & $0.961 \pm 0.081$ & $0.921 \pm 0.017$ & $0.941 \pm 0.093$ & $0.981 \pm 0.038$ \\
\hline 3D-UNet [10] & $0.920 \pm 0.108$ & $\underline{0.964} \pm \underline{0.038}$ & $0.880 \pm 0.133$ & $0.922 \pm 0.012$ & $0.981 \pm 0.039$ \\
\hline ResNet50 [9] & $\underline{0.943} \pm \underline{0.014}$ & $\underline{0.935} \pm \underline{0.018}$ & $\underline{0.945} \pm \underline{0.017}$ & $0.940 \pm 0.029$ & $\underline{0.982 \pm \underline{0.048}}$ \\
\hline Proposed Model & $\underline{0.944} \pm \underline{0.089}$ & $\underline{0.965} \pm \underline{0.085}$ & $0.935 \pm 0.052$ & $\underline{0.948} \pm \underline{0.051}$ & $\underline{0.983} \pm \underline{0.127}$ \\
\hline
\end{tabular}

TABLE 4: Performance analysis of the proposed semi-supervised model, Han et al. [19], 3D-UNet [10], ResNet50 [9] and Wang et al. [18] for COVID-19 detection on the IEEE CCAP data set [37] [One sided non-parametric two sample KS test [44] with $\alpha=0.05$ significance level has been conducted and marked underlined.]

\begin{tabular}{llllll}
\hline Model & Accuracy & Precision & Recall & $F 1-$ score & AUC \\
\hline Wang et al. [18] & $0.967 \pm 0.125$ & $0.961 \pm 0.027$ & $0.960 \pm 0.014$ & $0.961 \pm 0.053$ & $0.949 \pm 0.088$ \\
\hline ResNet50 $[9]$ & $\underline{0.984} \pm \underline{0.015}$ & $0.983 \pm 0.032$ & $\underline{0.986} \pm \underline{0.023}$ & $\underline{0.985} \pm \underline{0.023}$ & $0.968 \pm 0.047$ \\
\hline 3D-UNet [10] & $\underline{0.979} \pm 0.081$ & $0.974 \pm 0.018$ & $\underline{0.915} \pm 0.034$ & $0.979 \pm 0.015$ & $0.971 \pm 0.019$ \\
\hline Han et al. [19] & $\underline{0.985} \pm \underline{0.019}$ & $0.982 \pm 0.017$ & $\underline{0.987} \pm \underline{0.016}$ & $\underline{0.985} \pm \underline{0.016}$ & $0.973 \pm 0.032$ \\
\hline Proposed Model & $\underline{\underline{0.984}} \pm \underline{0.052}$ & $\underline{0.986} \pm \underline{0.163}$ & $0.985 \pm 0.071$ & $0.983 \pm 0.011$ & $\underline{0.978} \pm \underline{0.072}$ \\
\hline
\end{tabular}

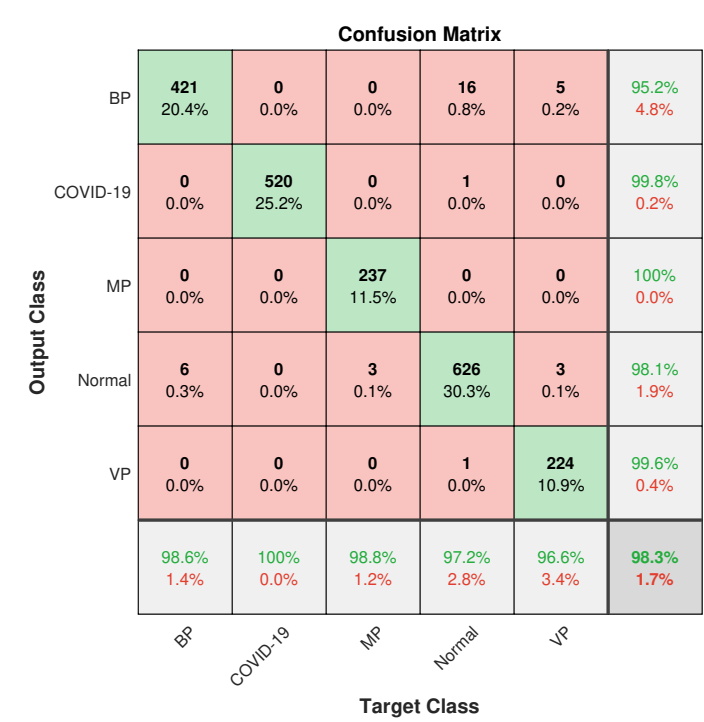

FIGURE 14: Confusion matrix for the accuracy of prediction of Bacterial Pneumonia (BP), COVID-19, Mycoplasma Pneumonia (MP), Normal lung, and Viral Pneumonia (VP) using the proposed semi-supervised shallow neural network model on IEEE CCAP data set [37] and inputs in quantum formalism thereby enabling faster convergence of the network states owing to reduction in computation. This intrinsic property of the PQIS-Net model yields precise and time efficient segmentation in real-time, which is evident from the results demonstrated in the experimental section. In spite being a semi-supervised model, the suggested semi-supervised shallow neural network has attained better stability on the outcome as it is evident from the higher values of precision and AUC compared with the state of art best methods specially in COVID-19 and Mycoplasma Pneumonia screening. It may be noted that ResNet50 and 3D-UNet marginally outperform the proposed PQIS-Net in lung CT image segmentation tasks in terms of dice similarity. This is due to the fact that the proposed PQISNet is a fully self-supervised neural network model based on pixel intensity based features. However, performance of the proposed semi-supervised model is very close to ResNet50 and Han et al. (statistically similar) and outperforms all the best published works in terms of Precision and AUC for COVID-19 diagnosis. In addition to this, the lower computational complexity and lesser resources required to implement the proposed semi-supervised framework make it a notable significant contribution in the field of semi-supervised or 
weakly supervised learning paradigms. Thus, the proposed parallel semi-supervised shallow learning model serves as an inspiration for promoting a potential alternative to the deep supervised learning frameworks for lung CT image segmentation for automatic COVID-19 diagnosis as well as for automatic medical image segmentation in various applications with limited labelled data sets. Moreover, our lightweighted semi-supervised model can be employed in any application setting (eg. medical IoT devices) right away where, the deep learning models face serious obstacles. It remains to investigate the performance of lung CT segmentation using the optimized version of PQIS-Net followed by classification with adaptive patch sizes. The authors are currently engaged in this direction.

\section{APPENDIX. CONVERGENCE ANALYSIS OF THE} PQIS-NET MODEL

The convergence of the network states in PQIS-Net is guided by the error or loss function and the segmented output is obtained once the network stabilizes. The coherent network error cum loss function is introduced in the PQIS-Net, is evaluated as follows.

$\zeta(\omega, \gamma)=\frac{1}{2} \sum_{k}^{\mathcal{B}} \sum_{i}^{m \times n} \sum_{j}^{\mathcal{N}}\left[\varphi^{\iota+1, k}\left(\omega_{i, j}^{\iota+1, k}, \gamma_{i}^{\iota+1, k}\right)-\varphi^{\iota, k}\left(\omega_{i, j}^{\iota, k}, \gamma_{i}^{\iota, k}\right)\right]^{2}$

where, $\varphi^{\iota, k}\left(\omega_{i, j}^{\iota, k}, \gamma_{i}^{\iota, k}\right)$ is the weighted inter-connection at a particular epoch $\iota$ and is linearly dependent on $\omega$ and $\gamma$. $\mathcal{B}$ is the batch size of the constituent PQIS-Net. The loss function $\zeta(\omega, \gamma)$ is guided by the phase or angles $\omega$ and $\gamma$. Each entry in the weighted inter-connection matrix in between the successive constituent layers in the PQIS-Net architecture is updated using rotation gate as follows.

$$
\begin{aligned}
\left|\varphi^{l+1, k}\right\rangle & =\left(\begin{array}{cc}
\cos \triangle \omega^{\iota, k} & -\sin \triangle \omega^{\iota, k} \\
\sin \triangle \omega^{\iota, k} & \cos \triangle \omega^{\iota, k}
\end{array}\right)\left|\varphi^{l, k}\right\rangle \\
\left|\xi^{l+1, k}\right\rangle & =\left(\begin{array}{cc}
\cos \triangle \gamma^{l, k} & -\sin \triangle \gamma^{l, k} \\
\sin \triangle \gamma^{l, k} & \cos \triangle \gamma^{l, k}
\end{array}\right)\left|\xi^{l, k}\right\rangle
\end{aligned}
$$

where,

$$
\omega^{l+1, k}=\omega^{\iota, k}+\triangle \omega^{\iota, k}
$$

and

$$
\gamma^{l+1, k}=\gamma^{l, k}+\triangle \gamma^{l, k}
$$

Hence, Equations 19 and 20 measure the change in phase or angles $\triangle \omega^{\iota, k}$ and $\triangle \gamma^{l, k}$, respectively for $k^{t h}$ constituent parallel network in PQIS-Net at epoch $\iota$. Consider

$$
\begin{aligned}
& \mathcal{W}^{\iota, k}=\omega^{\iota, k}-\overline{\omega^{\iota, k}} \\
& \mathcal{A}^{\iota, k}=\gamma^{\iota, k}-\overline{\gamma^{\iota, k}}
\end{aligned}
$$

and

$$
\begin{aligned}
& \mathcal{V}^{\iota, k}=\omega^{\iota+1, k}-\omega^{\iota, k}=\mathcal{W}^{\iota+1, k}-\mathcal{W}^{\iota, k} \\
& \mathcal{M}^{\iota, k}=\gamma^{\iota+1, k}-\gamma^{\iota, k}=\mathcal{A}^{\iota+1, k}-\mathcal{A}^{\iota, k}
\end{aligned}
$$

Hence, the optimal phase or angles for weighted inter-connection and the corresponding activation are $\overline{\omega^{\iota, k}}$ and $\overline{\gamma^{\iota, k}}$, respectively. Differentiation of the loss function $\zeta(\omega, \gamma)$ with respect to $\omega_{i j}^{\iota, k}, \gamma_{i}^{\iota, k}$ gives

$$
\begin{gathered}
\frac{\partial \zeta(\omega, \gamma)}{\partial \omega_{i j}^{\iota, k}}=\frac{2}{m n} \sum_{k=1}^{\mathcal{B}} \sum_{i=1}^{m \times n} \sum_{j=1}^{\mathcal{N}} \Delta \varphi_{i j}^{\iota, k}\left(\omega_{i j}^{\iota, k}, \gamma_{i}^{\iota, k}\right) \\
{\left[\frac{\partial \varphi_{i j}^{\iota+1, k}\left(\omega_{i j}^{\iota+1, k}, \gamma_{i}^{\iota+1, k}\right)}{\partial \omega_{i j}^{\iota, k}}-\frac{\partial \varphi_{i j}^{\iota, k}\left(\omega_{i j}^{\iota, k}, \gamma_{i}^{\iota, k}\right)}{\partial \omega_{i j}^{\iota, k}}\right]}
\end{gathered}
$$

$$
\begin{gathered}
\frac{\partial \zeta(\omega, \gamma)}{\partial \gamma_{i}^{\iota, k}}=\frac{2}{m n} \sum_{k=1}^{\mathcal{B}} \sum_{i=1}^{m \times n} \sum_{j=1}^{\mathcal{N}} \Delta \varphi_{i j}^{\iota, k}\left(\omega_{i j}^{\iota, k}, \gamma_{i}^{\iota, k}\right) \\
{\left[\frac{\partial \varphi_{i j}^{\iota+1, k}\left(\omega_{i j}^{\iota+1, k}, \gamma_{i}^{\iota+1, k}\right)}{\partial \gamma_{i}^{\iota, k}}-\frac{\partial \varphi_{i j}^{\iota, k}\left(\omega_{i j}^{\iota, k}, \gamma_{i}^{\iota, k}\right)}{\partial \gamma_{i}^{\iota, k}}\right]}
\end{gathered}
$$

where

$$
\triangle \varphi_{i j}^{\iota, k}\left(\omega_{i j}^{\iota, k}, \gamma_{i}^{\iota, k}\right)=\left|\varphi_{i j}^{\iota+1, k}\left(\omega_{i j}^{\iota+1, k}, \gamma_{i}^{\iota+1, k}\right)-\varphi_{i j}^{\iota, k}\left(\omega_{i j}^{\iota, k}, \gamma_{i}^{\iota, k}\right)\right|
$$

The changes in phase or angles are designated as $\Delta \omega_{i j}^{\iota, k}$ and $\Delta \gamma_{i j}^{\iota, k}$ for the rotation gate involved in updating the weighted matrix and its corresponding activation as follows.

$$
\begin{aligned}
\Delta \omega_{i j}^{\iota, k} & =-\mathcal{X}_{i j}^{\iota, k}\left\{\frac{\partial \zeta(\omega, \gamma)}{\partial \omega_{i j}^{\iota, k}} \zeta(\omega, \gamma)\right\}^{\frac{1}{\iota}} \\
\triangle \gamma_{i}^{\iota, k} & =-\kappa_{i}^{\iota, k}\left\{\frac{\partial \zeta(\omega, \gamma)}{\partial \gamma_{i}^{\iota, k}} \zeta(\omega, \gamma)\right\}^{\frac{1}{\iota}}
\end{aligned}
$$

Here, $\mathcal{X}_{i j}^{\iota, k}$ and $\kappa_{i}^{\iota, k}$ correspond to the learning rates in updating the angle of rotations in inter-connection matrix and its activation in $k^{t h}$ parallel network in PQIS-Net at epoch $\iota$. These are measured as follows.

$$
\mathcal{X}_{i j}^{\iota, k}=\mu_{i}^{\iota, k}-\mu_{i j}^{\iota, k} \forall j=1,2 \ldots \mathcal{N}
$$

and

$$
\kappa_{i}^{\iota, k}=\left(\sum_{j} \mu_{i, j}^{\iota, k}\right) \forall j=1,2 \mathcal{N}
$$

To show the super-linearly convergence of PQIS-Net, the following conditions on the sequences $\left\{\omega^{\iota, k}\right\}$ and $\left\{\gamma^{\iota, k}\right\}$ should be imposed [45].

$$
\lim _{\iota \rightarrow \infty} \frac{\left\|\omega^{\iota+1, k}-\overline{\omega^{\iota, k}}\right\|}{\left\|\omega^{\iota, k}-\overline{\omega^{\iota, k}}\right\|} \leq 1
$$

and

$$
\left\|\mathcal{W}^{\iota+1, k}\right\|=O\left\|\mathcal{V}^{\iota, k}\right\|
$$

Also,

$$
\lim _{\iota \rightarrow \infty} \frac{\left\|\gamma^{\iota+1, k}-\overline{\gamma^{\iota, k}}\right\|}{\left\|\gamma^{\iota, k}-\overline{\gamma^{\iota, k}}\right\|} \leq 1
$$

and

$$
\left\|\mathcal{A}^{\iota+1, k}\right\|=O\left\|\mathcal{M}^{\iota, k}\right\|
$$

Now, according to Thaler formula

$$
\begin{aligned}
& \zeta\left(\omega^{\iota+1, k}, \gamma^{\iota+1, k}\right)-\zeta\left(\omega^{\iota, k}, \gamma^{\iota, k}\right) \\
& {\left[\begin{array}{cc}
\triangle \omega_{i j}^{\iota, k} & \Delta \gamma_{i}^{\iota, k}
\end{array}\right]\left[\begin{array}{c}
\frac{\partial \zeta\left(\omega^{\iota, k}, \gamma^{\iota, k}\right)}{\partial \omega_{i j}^{,, k}} \\
\frac{\partial \zeta\left(\omega^{\iota, k}, \gamma^{\iota, k}\right)}{\partial \gamma_{i}^{\iota, k}}
\end{array}\right]+O\left[\begin{array}{ll}
\| \Delta \omega_{i j}^{\iota, k} & \left.\Delta \gamma_{i}^{\iota, k} \|\right]
\end{array}\right]} \\
& \approx\left[\left\{-\mathcal{X}_{i j} \frac{\partial \zeta\left(\omega^{\iota, k}, \gamma^{\iota, k}\right)}{\partial \omega_{i j}^{\iota, k}}\right\}^{2}+\left\{-\kappa_{i} \frac{\partial \zeta\left(\omega^{\iota, k}, \gamma^{\iota, k}\right)}{\partial \gamma_{i j}^{\iota, k}}\right\}^{2}\right]\left\{\zeta\left(\omega^{\iota, k}, \gamma^{\iota, k}\right)\right\}^{\frac{1}{\iota, k}}
\end{aligned}
$$

Here, it is evident that $\left(\zeta\left(\omega^{\iota+1, k}, \gamma^{\iota+1, k}\right)-\zeta\left(\omega^{\iota, k}, \gamma^{\iota, k}\right)\right) \leq 0$ and monotonically decreasing behavior of the given sequences $\left\{\omega^{\bar{\iota}, k}\right\}$ and $\left\{\gamma^{\iota, k}\right\}$ are as follows.

$$
\lim _{l \rightarrow \infty} \zeta\left(\omega^{\iota, k}, \gamma^{\iota, k}\right)=\left(\overline{\omega^{\iota, k}}, \overline{\gamma^{\iota, k}}\right)
$$

and

$$
\lim _{l \rightarrow \infty} \frac{\left\|\zeta\left(\omega^{\iota+1, k}, \gamma^{\iota+1, k}\right)-\left(\overline{\omega^{\iota, k}}, \overline{\gamma^{\iota, k}}\right)\right\|}{\left\|\zeta\left(\omega^{\iota, k}, \gamma^{\iota, k}\right)-\left(\overline{\omega^{\iota, k}}, \overline{\gamma^{\iota, k}}\right)\right\|} \leq 1
$$

The loss curve of the suggested PQIS-Net is provided in Figure 15. The convergence of the proposed semi-supervised network architecture (PQISNet followed by FC layers) is demonstrated during training and validation in the following Fig 16. It is evident from the experiments that the suggested network converges at epoch \#10. 
2
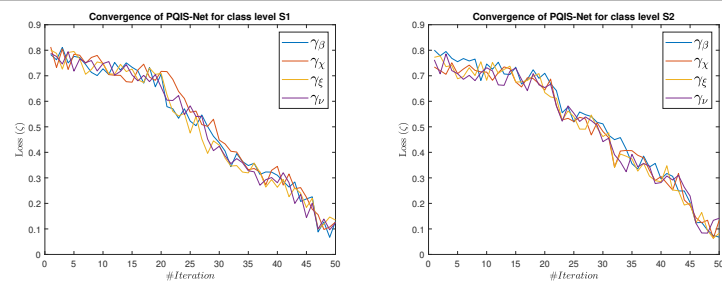

(a) Class Bondary1 (S1)

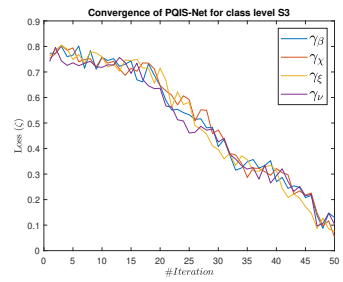

(c) Class Bondary3 (S3) (b) Class Bondary2 (S2)

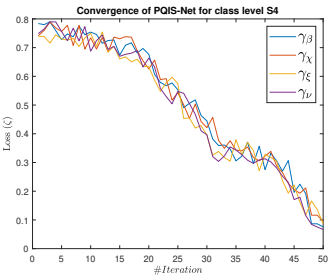

(d) Class Bondary4 (S4)
FIGURE 15: Convergence of the proposed PQIS-Net for four different activation with four distinct class boundaries and using the data set [37]

\section{REFERENCES}

[1] W. H. O., "Coronavirus disease (COVID-19) pandemic.", https://www. who. int/emergencies/diseases/novel-coronavirus-2019., 2020.

[2] W. H. O., "https://www.who.int/publications/m/item/weeklyepidemiological-update-10-november-2020," who.int/docs/default-source/coronaviruse/weekly-updates/ wou-9-september-2020-cleared.pdf?sfvrsn=d39784f7_2, https://www. September 11, 2020.

[3] L. Fan, D. Li, H. Xue, L. Zhang, Z. Liu, B. Zhang et al., "Progressand prospect on imaging diagnosis of COVID-19", Chinese Journal of Academic Radiology, vol. 3, pp. 4—13, 2020, DOI: https://doi.org/10.1007/ s42058-020-00031-5.

[4] T. Ai, Z. Yang, H. Hou, C. Zhan, C. Chen, W. Lv, Q. Tao, Z. Sun, and L. Xia, "Correlation of chest CT and RT-PCR testing in coronavirus disease 2019 (COVID-19) in china: A report of 1014 cases", 2020, https://doi.org/ 10.1148/radiol.2020200642.

[5] D. Ardila, A. P. Kiraly, S. Bharadwaj, B. Choi, J. J. Reicher, L. Peng, et al., "End-to-end lung cancer screening with three-dimensional deep learning on low-dose chest computed tomography," Nature Medicine, vol. 25, pp. 954-961, 2019, DOI: https://doi.org/10.1038/s41591-019-0447-x.

[6] N. Coudray, P. S. Ocampo, T. Sakellaropoulos, et al., "Classification and mutation prediction from non-small cell lung cancer histopathology images using deep learning," Nature Medicine, vol. 24, pp. 1559--1567, 2018, DOI: https://doi.org/10.1038/s41591-018-0177-5.

[7] F. Liao, M. Liang, Z. Li, X. Hu, and S. Song, "Evaluate the Malignancy of Pulmonary Nodules using the 3-D Deep Leaky Noisy-OR Network," IEEE Transactions on Neural Networks and Learning Systems, vol.30, no.11, 2019, DOI: 10.1109/TNNLS.2019.2892409.

[8] H. Zhu, H. Zhao, C. Song, Z. Bian, Y. Bi, T. Liu, X. He, D. Yang, and W. Cai, "MR-Forest: A Deep Decision Framework for False Positive Reduction in Pulmonary Nodule Detection," IEEE Journal of Biomedical and Health Informatics, vol. 24, no. 6, 2020, DOI: 10.1109/JBHI.2019.2947506.

[9] K. He, X. Zhang, S. Ren, and J. Sun, "Deep Residual Learning for Image Recognition," 2016 IEEE Conference on Computer Vision and Pattern Recognition (CVPR), 2016, DOI: 10.1109/CVPR.2016.90.

[10] J. Ma et al.,"Towards Efficient COVID-19 CT Annotation: A Benchmark for Lung and Infection Segmentation,"arXiv:2004.12537, 2020.

[11] L. Li et al., "Artificial Intelligence Distinguishes COVID-19 from Community Acquired Pneumonia on Chest CT,", Radiology, 2020, DOI: https: //doi.org/10.1148/radiol.2020200905.

[12] D. Singh, V. Kumar, Vaishali, and M. Kaur, "Classification of COVID19 patients from chest CT images using multi-objective differential evolution-based convolutional neural networks," European Journal of Clinical Microbiology and Infectious Diseases, 2020, DOI: https://doi.org/10.1007/ s10096-020-03901-z.
[13] S. Roy et al., "Deep learning for classification and localization of COVID19 markers in point-of-care lung ultrasound," IEEE Transactions on Medical Imaging, 2020, DOI: 10.1109/TMI.2020.2994459.

[14] S. Wang et al., "A deep learning algorithm using CT images to screen for coronavirus Disease (COVID-19)," medRxiv, 2020, DOI: https://doi.org/ 10.1101/2020.02.14.20023028.

[15] H. Kang et al.,"Diagnosis of Coronavirus Disease 2019 (COVID-19) with Structured Latent Multi-View Representation Learning," IEEE Transactions on Medical Imaging, 2020, DOI:http://dx.doi.org/10.1109/TMI.2020. 2992546.

[16] C. Zheng, X. Deng, Q. Fu, and Q. Zhou, "Deep Learning-based Detection for COVID-19 from Chest CT using Weak Label," MedRxiv, 2020, DOI: https://doi.org/10.1101/2020.03.12.20027185.

[17] Q. Yan et al., "COVID-19 Chest CT Image Segmentation -A Deep Convolutional Neural Network Solution," https://arxiv.org/abs/2004.10987, 2020.

[18] J. Wang, Y. Bao, Y. Wen, H. Lu, H. Luo, Y. Xiang, X. Li, and C. Liu, "Prior-Attention Residual Learning for More Discriminative COVID-19 Screening in CT Images," IEEE Transactions on Medical Imaging, 2020, DOI: 10.1109/TMI.2020.2994908.

[19] Z. Han, B. Wei, Y. Hong, T. Li, J. Cong, X. Zhu, H. Wei, and W. Zhang, "Accurate Screening of COVID-19 Using Attention-Based Deep 3D Multiple Instance Learning," IEEE Transactions on Medical Imaging, vol. 39, no. 8, August 2020, DOI: 10.1109/TMI.2020.2996256.

[20] D. Konar, S. Bhattacharyya, T. K. Gandhi, and B. K. Panigrahi, "A quantum-inspired self-supervised Network model for automatic segmentation of brain MR images," Applied Soft Computing, vol. 93, 2020, DOI: https://doi.org/10.1016/j.asoc.2020.106348.

[21] D. Konar, S. Bhattacharyya, and B. K. Panigrahi, "QIBDS Net: A Quantum-Inspired Bi-Directional Self-supervised Neural Network Architecture for Automatic Brain MR Image Segmentation," Proc. 8th International Conference on Pattern Recognition and Machine Intelligence (PReMI 2019), vol. 11942, pp. 87-95, 2019, DOI: https://doi.org/10.1007/ 978-3-030-34872-4_64.

[22] D. Konar, S. Bhattacharyya, S. Dey, and B. K. Panigrahi, "Opti-QIBDS Net: A Quantum-Inspired Optimized Bi-Directional Self-supervised Neural Network Architecture for Automatic Brain MR Image Segmentation," Proc. 2019 IEEE Region 10 Conference (TENCON), pp. 761-766, 2019, DOI: 10.1109/TENCON.2019.8929585.

[23] A. Ghosh, N. R. Pal, and S. K. Pal, "Self organization for object extraction using a multilayer neural network and fuzziness measures," IEEE Transactions on Fuzzy Systems, vol. 1, no.1, pp. 54-68, 1993, DOI: 10.1109/TFUZZ.1993.390285.

[24] S. Bhattacharyya, P. Dutta, and U. Maulik, "Binary object extraction using bi-directional self-organizing neural network (BDSONN) architecture with fuzzy context sensitive thresholding," Pattern Anal Applic., vol. 10, pp. 345-360, 2007, DOI: https://doi.org/10.1007/s10044-007-0072-z.

[25] S. Bhattacharyya, P. Dutta, and U. Maulik, "Multilevel image segmentation with adaptive image context based thresholding", Applied Soft Computing, vol. 11, no.1, pp. 946-962, 2011, DOI: https://doi.org/10.1016/j. asoc.2010.01.015.

[26] D. Konar, S. Bhattacharya, U. Chakraborty, T. K.Gandhi, and B. K. Panigrahi, "A quantum parallel bi-directional self-organizing neural network (QPBDSONN) architecture for extraction of pure color objects from noisy background", Proc. IEEE International Conference on Advances in Computing, Communications and Informatics (ICACCI),2016, pp. 1912-1918, 2016, DOI: 10.1109/ICACCI.2016.7732330.

[27] D. Konar, S. Bhattacharya, B. K. Panigrahi, and K. Nakamatsu, "A quantum bi-directional self-organizing neural network (QBDSONN) architecture for binary object extraction from a noisy perspective," Applied Soft Computing, vol.46, pp. 731-752, 2016, DOI: https://doi.org/10.1016/j.asoc. 2015.12.040.

[28] S. Bhattacharyya, P. Pal and S. Bhowmick, "Binary Image Denoising Using a Quantum Multilayer Self Organizing Neural Network," Applied Soft Computing, vol.24, pp. 717-729, 2014, https://doi.org/10.1016/j.asoc. 2014.08.027.

[29] S. Dey, I. Saha, S. Bhattacharyya, U. Maulik, Multi-level thresholding usingquantum inspired meta-heuristics, Knowledge Based System 67 (2014) $373-400$.

[30] N. Otsu, "A threshold selection method from gray level histograms," IEEE Transactions on Systems, Man, and Cybernetics, vol. 9, no. 1, pp. 62-66, 1979.

[31] L. Hou, D. Samaras, T. M. Kurc, Y. Gao, J. E. Davis, and J. H. Saltz, "Patch-based Convolutional Neural Network for Whole Slide Tissue Image 


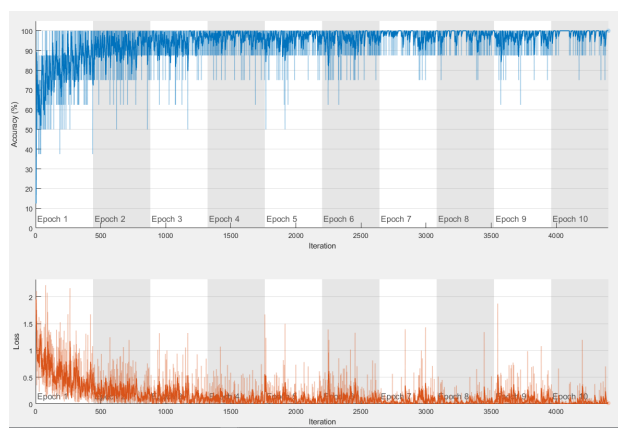

(a) \#1-fold cross validation

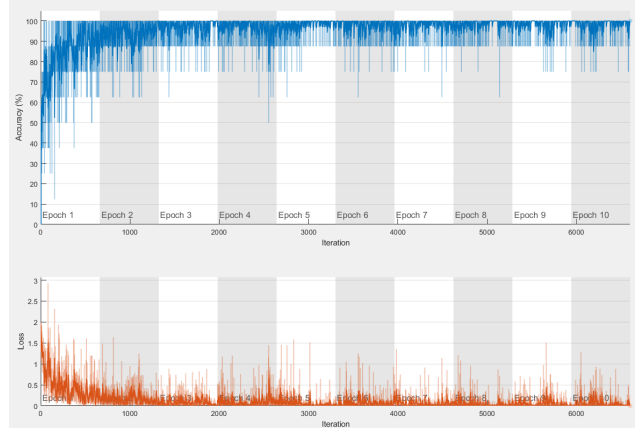

(c) \#3-fold cross validation

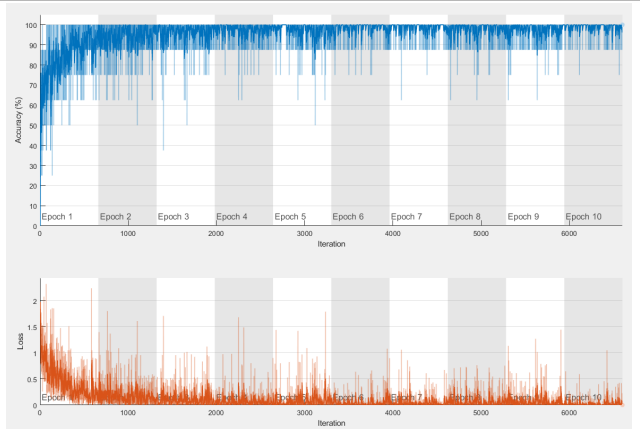

(b) \#2-fold cross validation

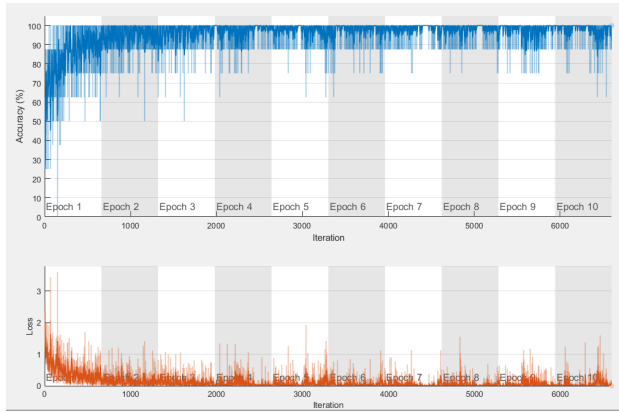

(d) \#4-fold cross validation

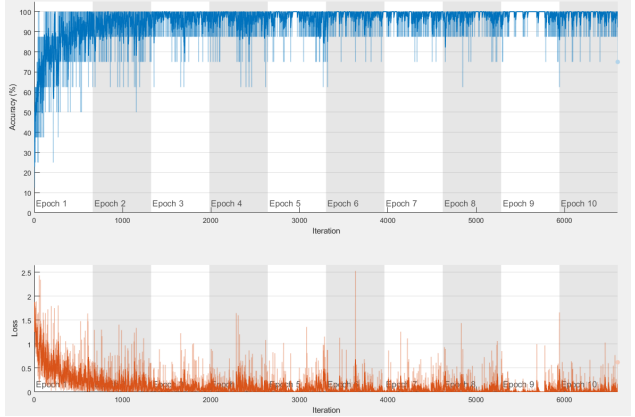

(e) \#5-fold cross validation

FIGURE 16: Convergence of the proposed semi-supervised shallow neural learning model allowing maximum 10 epochs during training and validation with an initial learning rate $=0.01$ using the IEEE CCAP data set [37]

Classification," Proc. of the 2016 IEEE Conference on Computer Vision and Pattern Recognition (CVPR), 2016, DOI: 10.1109/CVPR.2016.266.

[32] A. Sharmaa, X. Liua, X. Yangb, and D. Shic, "A patch-based convolutional neural network for remote sensing image classification," Neural Networks, vol. 95, pp. 19-28,2017, DOI: https://doi.org/10.1016/j.neunet.2017.07. 017.

[33] C. Kervrann, and J. Boulanger, "Unsupervised Patch-Based Image Regularization and Representation," Proc. of the 2006 European Conference on Computer Vision (ECCV), vol. 3954, pp. 555-567, DOI: https://doi.org/10. 1007/11744085_43

[34] Y. Oh, S. Park, and J. C. Ye, "Deep Learning COVID-19 Features on CXR using Limited Training Data Sets,", IEEE Transactions on Medical Imaging, 2020, DOI: 10.1109/TMI.2020.2993291.

[35] E. Soares, P. Angelov, S. Biaso, M. H. Froes, and D. K. Abe, "SARS-CoV-2 CT-scan dataset: A large dataset of real patients CT scans for SARS-CoV2 identification," medRxiv, 2020, DOI: https://doi.org/10.1101/2020.04.24 20078584.

[36] M. Jun et al., "COVID-19 CT Lung and Infection Segmentation Dataset (Version Verson 1.0) [Data set]," Zenodo.http://doi.org/10.5281/zenodo. 3757476, 2020.

[37] T. Yan, "CCAP," IEEE Dataport, 2020, [Online]., Available: http://dx.doi. org/10.21227/ccgv-5329. Accessed: August 07, 2020.
[38] R. Guerrero et al., "White matter hyperintensity and stroke lesion segmentation and differentiation using convolutional neural networks," NeuroImage: Clinical, vol. 17, pp. 918—934, 2018, DOI: https://doi.org/10.1016/j. nicl.2017.12.022.

[39] N. Otsu, "A Threshold Selection Method from Gray-Level Histograms," IEEE Transactions on Systems, Man, and Cybernetics, vol.9, no. 1, pp. 6266, 1979, DOI: 10.1109/TSMC.1979.4310076.

[40] F. Milletari, N. Navab, and S. A. Ahmadi, "V-Net: Fully convolutional neural networks for volumetric medical image segmentation," Proc.in 2016 Fourth IEEE International Conference on 3D Vision (3DV) 2016, pp. 565571, DOI: 10.1109/3DV.2016.79.

[41] F. Shan et al., "Lung Infection Quantification of COVID-19 in CT Images with Deep Learning," arXiv:2003.04655v3, 2020.

[42] C. Zhang, Z. Han, Y. Cui, H. Fu, J. T. Zhou, Q. Hu, and "CPM-Nets: Cross partial multi-view networks," In Proc. Advances in Neural Information Processing Systems, 2019, pp. 557-567.

[43] T. Fernando, H. Ghaemmaghami, S. Denman, S. Sridharan, N. Hussain, and C. Fookes, "Heart Sound Segmentation Using Bidirectional LSTMs With Attention", IEEE Journal of Biomedical and Health Informatics, vol.24, no.6, pp. 1601-1609, 2020, DOI: 10.1109/JBHI.2019.2949516

[44] M. H. Gail and S. B. Green, "Critical values for the one-sided two-sample Kolmogorov-Smirnov statistic," J. Am. Stat. Assoc., vol. 71, pp. 757-760, 


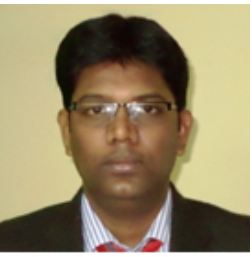

DEBANJAN KONAR (M'17) is currently pursuing $\mathrm{PhD}$ (Thesis submitted) from Indian Institute of Technology Delhi, New Delhi, India and also serving as a Research Assistant Professor in the Department of Computer Science and Engineering, Sikkim Manipal Institute of Technology, Sikkim, India. He completed his Bachelor in Engineering (CSE) from University of Burdwan, India in 2010, M. Tech (CSE) from National Institute of Technical Teachers' Training and Research (NITTTR), Kolkata, India in 2012. His fields of research are Deep Learning, Machine Learning, Quantum Computing, Medical Image Analysis. Mr. Konar has published several articles, conference proceedings, book chapters and edited books of international repute. He is a member of IEEE, ACM and IE and reviewer of several esteemed journals and International conferences.

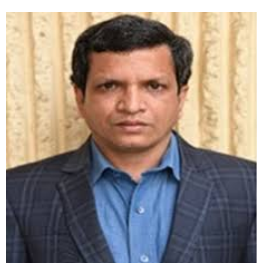

BIJAYA K. PANIGRAHI is working as Institute Chair Professor in the Department of Electrical Engineering, Indian Institute of Technology (IIT), Delhi, India. In addition, he is currently heading the Centre for Automotive Research and Tribology (CART), IIT Delhi. The research interest of Dr. Panigrahi is Biomedical imaging, Machine Intelligence and Evolutionary Computing. His research interest also includes digital signal processing techniques and its application for power quality monitoring and power system protection.He has served as the editorial board member/Associate Editor/Special Issue Guest Editor of different international journals. He is also associated with various international conferences in various capacities. He has published more than 150 research papers in various international and national journals including IEEE Transactions, Elsevier, Springer and conferences of international repute. He is also Fellow of Indian National Academy of Engineering (INAE).

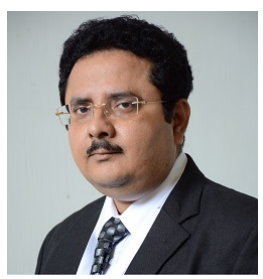

SIDDHARTHA BHATTACHARYYA (M'10SM'13) received the bachelor's degree in physics, and the bachelor's and master's degrees in optics and optoelectronics from the University of Calcutta, Kolkata, India, in 1995, 1998, and 2000, respectively, and the $\mathrm{Ph} . \mathrm{D}$. degree in computer science and engineering from Jadavpur University, Kolkata, in 2008.

$\mathrm{He}$ is currently serving as a Professor with the Department of Computer Science and Engineering, CHRIST (Deemed to be University), Bangalore, India. Prior to this, he served as the Principal of the RCC Institute of Information Technology, Kolkata. He also served as a Senior Research Scientist with the Faculty of Electrical Engineering and Computer Science, VSB Technical University of Ostrava, Ostrava, Czech Republic. He has coauthored five books, coedited 40 books, and has authored or co-authored more than 250 research publications in international journals and conference proceedings. He holds three patents. His research interests include soft computing, pattern recognition, multimedia data processing, hybrid intelligence, social networks, and quantum computing.

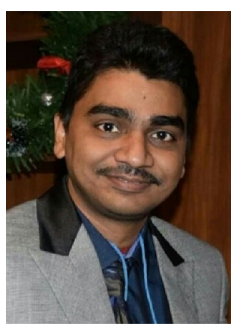

NILANJAN DEY is an Associate Professor in the Department of Computer Science and Engineering, JIS University, Kolkata, India. He is a visiting fellow of the University of Reading, UK. $\mathrm{He}$ is an Adjunct Professor of Ton Duc Thang University, Ho Chi Minh City, Vietnam. Previously, he held an honorary position of Visiting Scientist at Global Biomedical Technologies Inc., CA, USA (2012-2015). He was awarded his PhD from Jadavpur University in 2015. He has authored/edited more than 80 books with Elsevier, CRC Press, and Springer Nature, and published more than 300 papers. He is the Editor-in-Chief of the International Journal of Ambient Computing and Intelligence (IGI Global) Associated Editor of IEEE Access, and International Journal of Information Technology (Springer). He is the Series Co-Editor of Springer Tracts in Nature-Inspired Computing (Springer), Series Co-Editor of Advances in Ubiquitous Sensing Applications for Healthcare (Elsevier), Series Editor of Computational Intelligence in Engineering Problem Solving and Intelligent Signal Processing and Data Analysis (CRC). His main research interests include medical imaging, machine learning, computer aided diagnosis, data mining, etc. He is the Indian Ambassador of the International Federation for Information Processing-Young ICT Group and Senior member of IEEE.

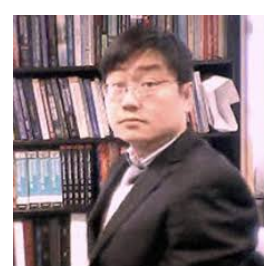

RICHARD JIANG is a Senior Lecturer (Associate Professor) in the School of Computing \& Communications at Lancaster University, UK. He is a Fellow of HEA, a Leverhulme Research Fellow, an EPSRC RISE Connector scientist, and an Associate Member of EPSRC College. Dr Jiang's research interest mainly resides in the fields of Artificial Intelligence, AI Ethics, Privacy and Security, Quantum AI, Neuronal Computation and Biomedical Image Analysis. His recent research has been supported by grants from EPSRC (EP/P009727/1), Leverhulme Trust (RF2019-492), Qatar Science Foundation (NPRP No.8-140-2-065) and other industry/international funders. He has supervised and co-supervised $12 \mathrm{PhD}$ students. He authored over 80 publications and was the lead editor of three Springer books. He served as a PC/Editorial member and a reviewer for various international conferences and journals. 


\title{
Supplementary Materials
}

\author{
Debanjan Konar, Bijaya K. Panigrahi, Siddhartha Bhattacharyya, \\ Nilanjan Dey, and Richard Jiang
}

I. Parallel Quantum-Inspired Bi-Directional Self-Supervision Algorithm

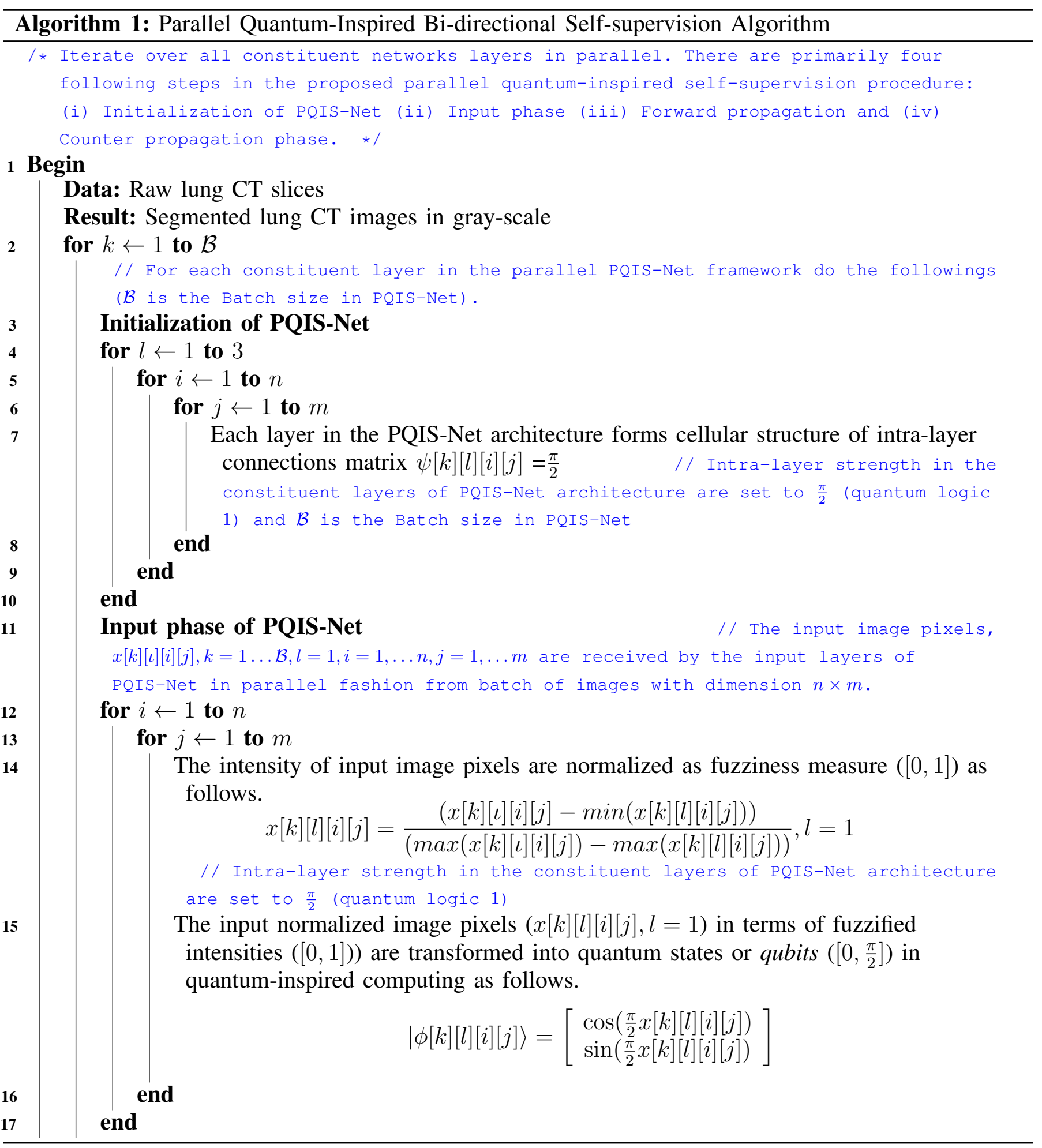




\section{Forward propagation phase in PQIS-Net}

for $l \leftarrow 1$ to 3

for $i \leftarrow 1$ to $n$

for $j \leftarrow 1$ to $m$

for $p \leftarrow-1$ to 1

for $q \leftarrow-1$ to 1

The rotation angle associated with every inter-linked weight in the weighted matrix between the layers in PQIS-Net architecture and the corresponding activation is evaluated as follows.

$$
\begin{aligned}
& \omega[k][l][i][j]=1-(\phi[k][l][i][j]-\phi[k][l][i+p][j+q]) \\
& \gamma[k][l][i]=2 \pi \times \sum_{j} \phi[k][l][i+p][j+q] \\
& \left|\varphi[k][l][i][j]\left[i^{\prime}\right]\right\rangle=\left[\begin{array}{l}
\cos \left(\frac{\pi}{2} \omega[k][l][i][j]\right) \\
\sin \left(\frac{\pi}{2} \omega[k][l][i][j]\right)
\end{array}\right] \\
& |\xi[k][l][i]\rangle=\left[\begin{array}{c}
\cos \gamma[k][l][i] \\
\sin \gamma[k][l][i]
\end{array}\right]
\end{aligned}
$$
quantum-inspired neuron and its $\mathcal{N}$-connected neighborhood pixels (neurons) at a particular constituent layer $l$ in a batch $k$ (parallel layers) are represented as $\phi[k][l][i][j]$ and $\phi[k][l][i+p][j+q]$, respectively in quantum formalism.

Each entry in the weighted inter-connection matrix in between the successive constituent layers in the PQIS-Net architecture is updated using rotation gate as follows.

$$
\begin{aligned}
& R(\omega[k][l][i][j])=\left[\begin{array}{cc}
\cos \left(\frac{\pi}{2} \omega[k][l][i][j]\right) & -\sin \left(\frac{\pi}{2} \omega[k][l][i][j]\right) \\
\sin \left(\frac{\pi}{2} \omega[k][l][i][j]\right) & \cos \left(\frac{\pi}{2} \omega[k][l][i][j]\right)
\end{array}\right] \\
& |\varphi[l+1][k][l][l+1][i][j]\rangle=R(\omega[k][l][i][j]) \times|\varphi[l][k][l][l+1][i][j]\rangle \\
& / / R(\omega[k][l][i][j]) \text { corresponds the rotation gate operation. The } \\
& \text { strength of a weighted inter-connection between two successive } \\
& \text { constituent layers } l \text { and } l+1 \text { in a batch } k \text { and at a particular } \\
& \text { epoch (l) in the PQIS-Net is } \varphi[l][k][l][l+1][i][j]
\end{aligned}
$$

The output of each quantum neuron at the constituent layers in PQIS-Net is evaluated as inner product of processed input matrix and weight matrix in quantum formalism guided by the Quantum Multi-level Sigmoidal activation function $\left(\sigma_{P Q I S-N e t}\right)$ as

$$
\begin{aligned}
& y[k][l+1][i][j]= \\
& \sum_{p=-1}^{1} \sum_{q=-1}^{1}[\sigma(y[k][l][i+p][j+q] * \varphi[l][k][l][l+1][i+p][j+q])] \\
& \text { // ' } * \text { ' designates the inner product operator which is defined as } \\
& \text { the sum of products of the entries of the two matrices input and } \\
& \text { weight in quantum formalism. }
\end{aligned}
$$




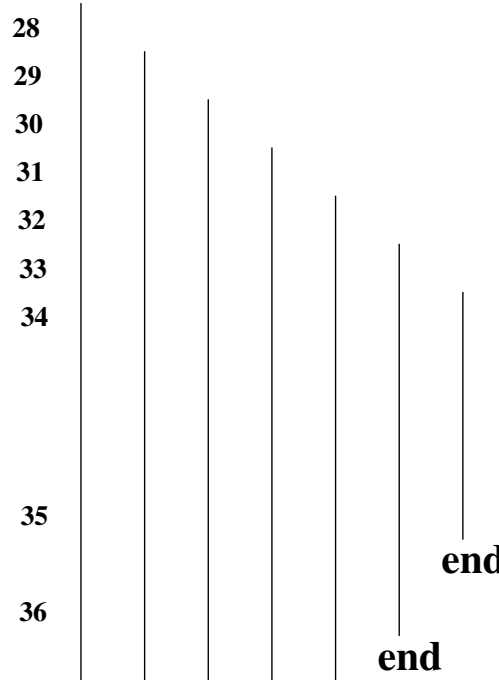

Quantum observation allows the imaginary or sin part to be computed as logic 1 as follows.

$$
\begin{aligned}
& z=y[k][l][i+p][j+q] * \varphi[l][k][l][l+1][i+p][j+q])= \\
& y[k][l][i+p][j+q] \times \sin (\omega[k][l][i][j]-\gamma[k][l][i])
\end{aligned}
$$

Here, $\sigma$ is defined as follows.

$$
\sigma\left(z ; \lambda_{\theta}, \mathcal{S}_{\theta}, \gamma\right)=\sum_{\theta=1}^{L} \frac{1}{\lambda_{\theta}+e^{-\nu\left(z-(\theta-1) \mathcal{S}_{\theta-1}-\gamma\right)}}
$$

and

$$
\lambda_{\theta}=\frac{\mathcal{N}_{s}[k][l][i][j]}{\mathcal{S}_{\theta}-\mathcal{S}_{\theta-1}}, \mathcal{S}_{\theta-1} \leq y[k][l][i][j] \leq \mathcal{S}_{\theta}, \theta=1, \ldots \ldots \mathcal{N}
$$

where,

$$
\mathcal{N}_{s}[k][l][i][j]=\sum_{p=-1}^{1} \sum_{q=-1}^{1} y[k][l][i+p][j+q]
$$

end

end

end 
do

The intermediate outputs at the constituent hidden layer are propagated in bi-directional fashion using steps 29,30,49 as follows.

Counter propagation phase in PQIS-Net

for $l \leftarrow 2$ to 3

$$
\begin{aligned}
& \text { for } i \leftarrow 1 \text { to } n \\
& \text { for } j \leftarrow 1 \text { to } m \\
& \quad \text { for } p \leftarrow-1 \text { to } 1 \\
& \quad \text { for } q \leftarrow-1 \text { to } 1
\end{aligned}
$$

The weighted matrix of inter-linked connections between the intermediate and output layers in the PQIS-Net architecture and its corresponding activation are set through updating rotation angles as follows.

$$
\begin{aligned}
& \begin{array}{c}
\omega[k][l][i][j]=1-(\phi[k][l][i][j]-\phi[k][l][i+p][j+q]) \\
\gamma[k][l][i]=2 \pi \times \sum_{j} \phi[k][l][i+p][j+q]
\end{array} \\
& \left|\varphi[k][l][i][j]\left[i^{\prime}\right]\right\rangle=\left[\begin{array}{l}
\cos \left(\frac{\pi}{2} \omega[k][l][i][j]\right) \\
\sin \left(\frac{\pi}{2} \omega[k][l][i][j]\right)
\end{array}\right] \\
& \qquad \xi[k][l][i]\rangle=\left[\begin{array}{l}
\cos \gamma[k][l][i] \\
\sin \gamma[k][l][i]
\end{array}\right] \\
& \text { quantum-inspired neuron and its } \mathcal{N} \text {-connected neighborhood } \\
& \text { pixels (neurons) at a particular constituent layer } l \text { in a } \\
& \text { batch } k \text { (parallel layers) are represented as } \phi[k][l][i][j] \text { and } \\
& \phi[k][l][i+p][j+q], \text { respectively for } \\
& k=1 \ldots \mathcal{B}, l=1,2,3, i=1, \ldots n, j=1, \ldots m, \text { and } p, q \in\{-1,1\} \text { in quantum }
\end{aligned}
$$

The weighted inter-connection matrix in between the successive constituent layers in the PQIS-Net architecture is updated using rotation gate as follows.

$$
\begin{aligned}
& |\varphi[\iota+1][k][\iota][l-1][i][j]\rangle=R(\omega[k][\iota][i][j]) \times|\varphi[\iota][k][\iota][l-1][i][j]\rangle \\
& \qquad / / \text { The strength of a weighted inter-connection between two } \\
& \text { successive constituent layers } l \text { and } l-1 \text { in a batch } k \text { and at a } \\
& \text { particular epoch }(\iota) \text { in the PQIS-Net is } \varphi[\iota][k][\iota][l-1][i][j] \text { for } \\
& k=1 \ldots \mathcal{B}, l=2,3, i=1, \ldots n, j=1, \ldots m
\end{aligned}
$$




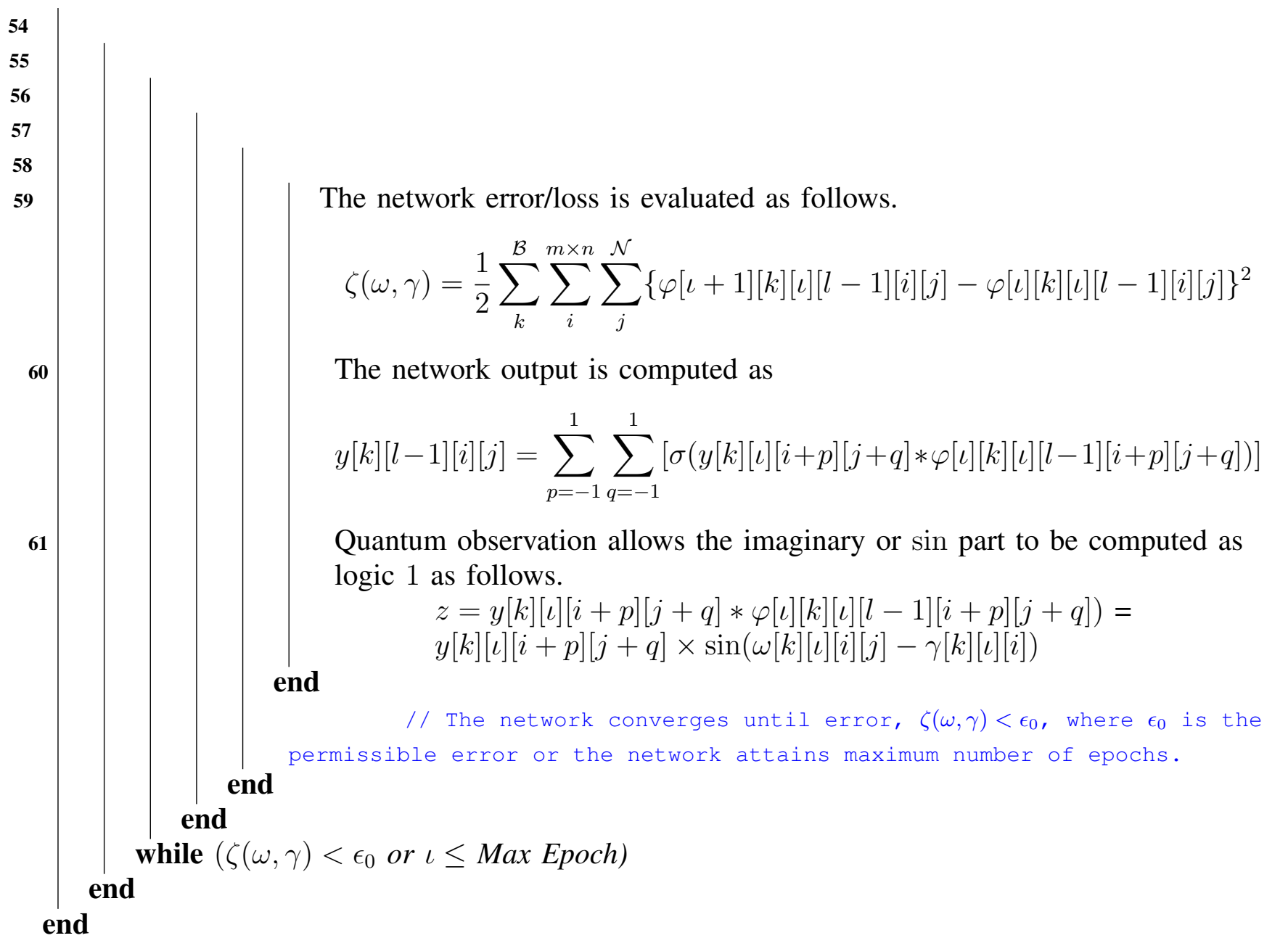




\section{PQIS-NET ASSISTED SEMI-SUPERVISED SHALLOW LEARNING FRAMEWORK}

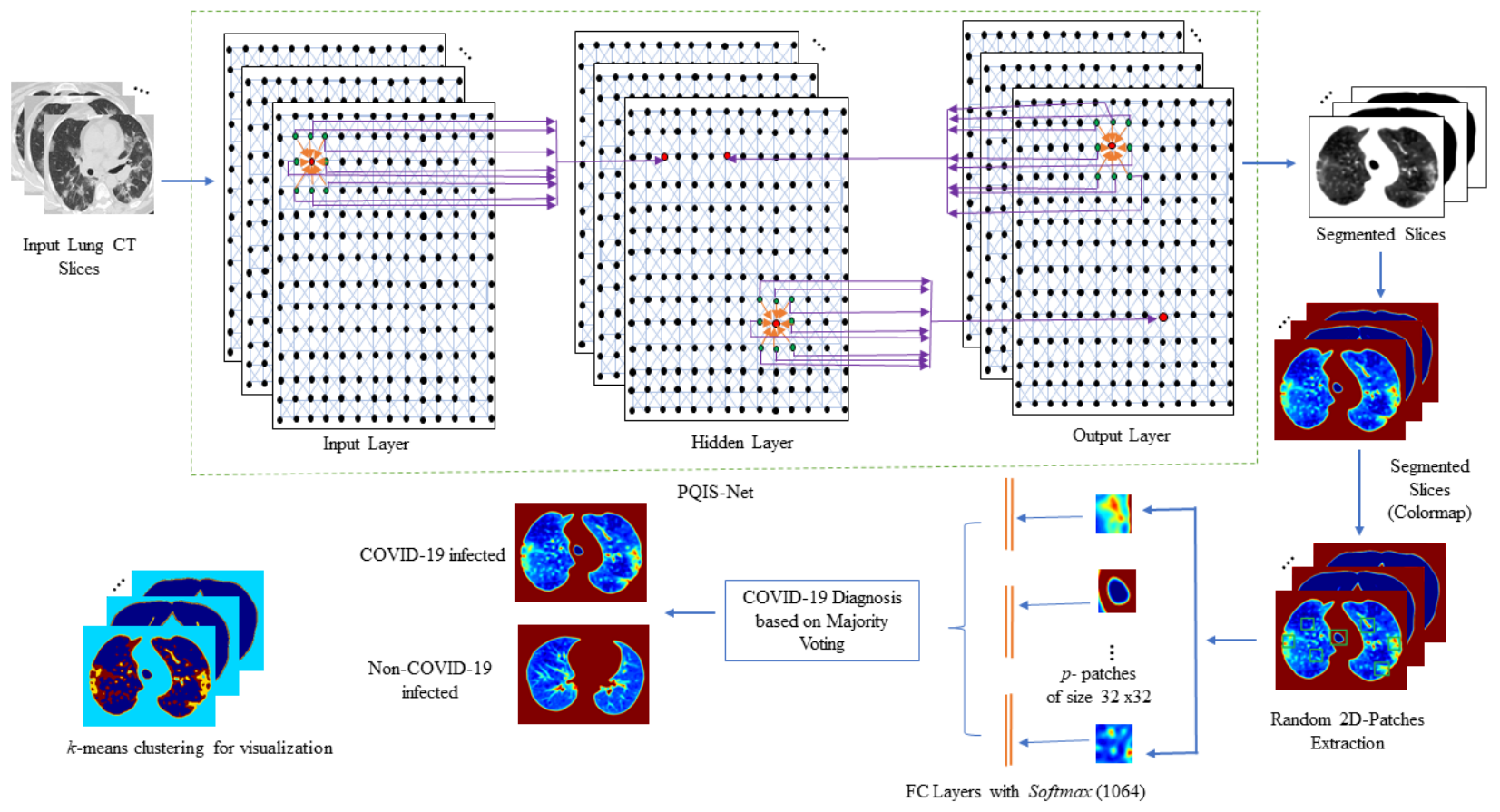

Fig. 1: A Parallel Quantum-Inspired Self-Supervised Network (PQIS-Net) assisted semi-supervised shallow learning framework for COVID-19 diagnosis (only three inter-layer connections are shown for clarity and gray-scale segmented slices are color mapped for better visibility).

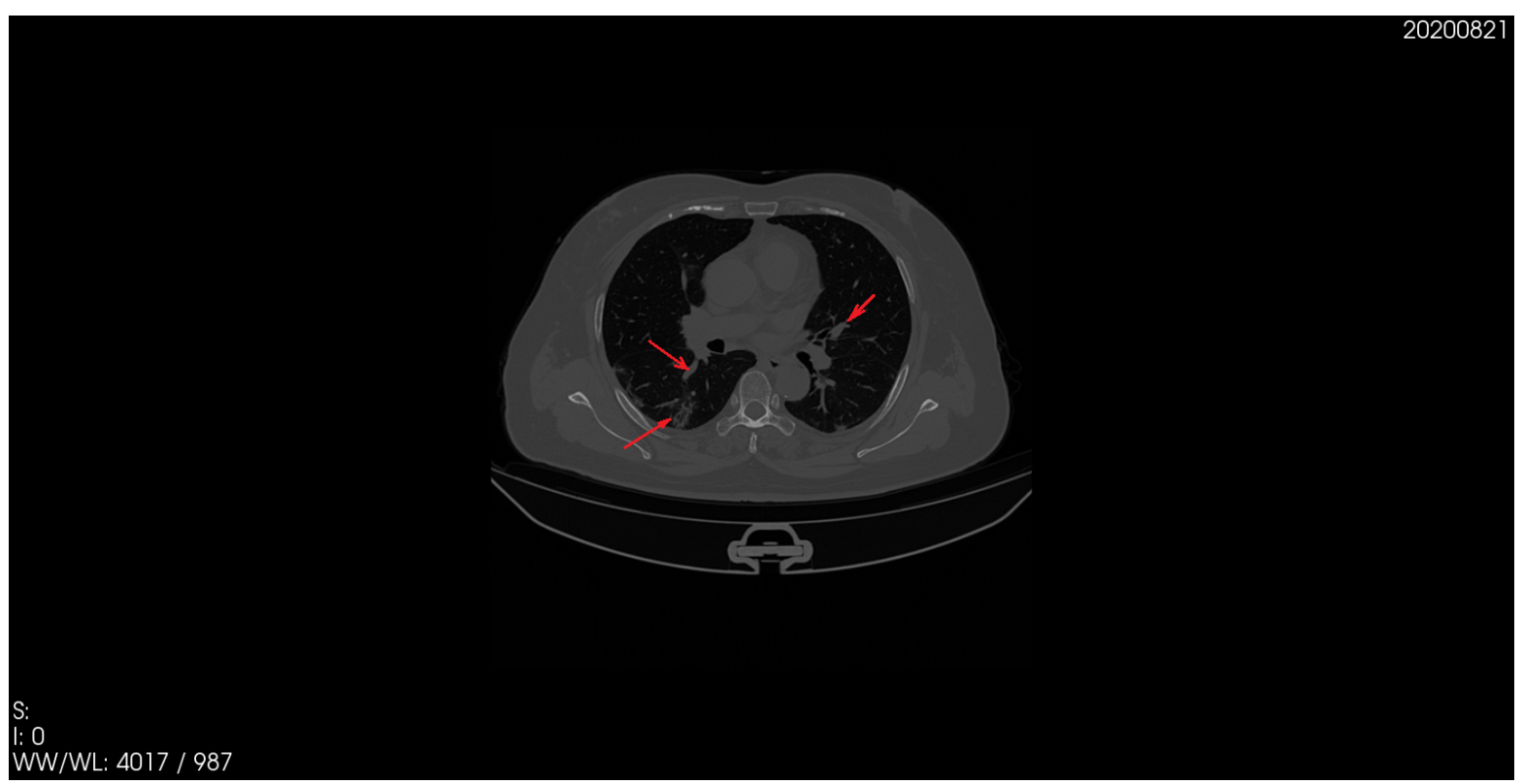

Fig. 2: Randomly Chosen input lung CT image slice\#001 - 171 [1] is shown in Lung Window (W/L: 4017/987) and infections are shown red arrow.

\section{REFERENCES}

[1] M. Jun et al., "COVID-19 CT Lung and Infection Segmentation Dataset (Version Verson 1.0) [Data set],” Zenodo.http://doi.org/10.5281/ zenodo.3757476, 2020. 


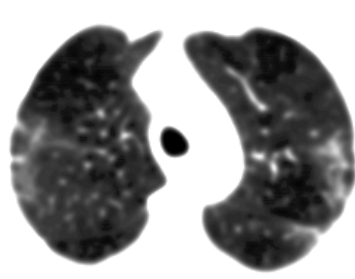

(a) \#1

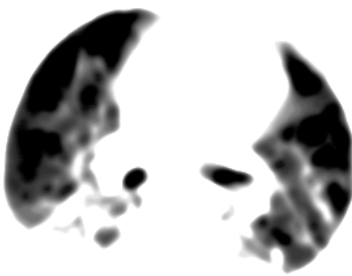

(e) \#242

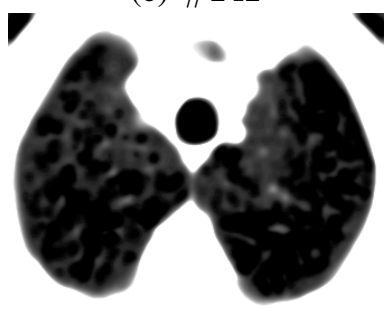

(i) $\# 3$
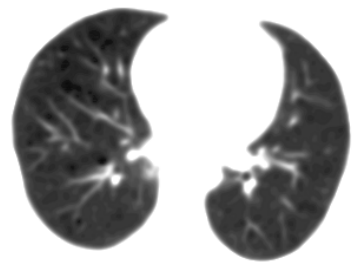

(m) \#290
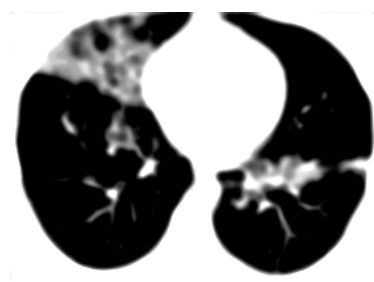

(b) $\# 27$

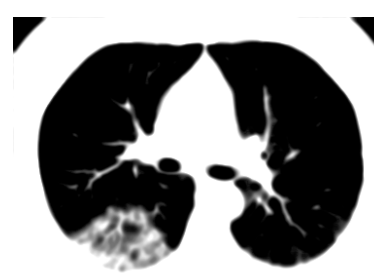

(f) $\# 343$

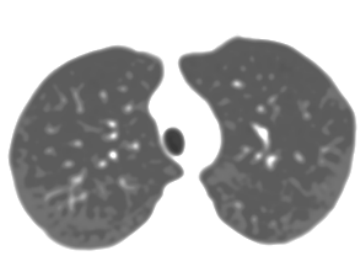

(j) $\# 28$
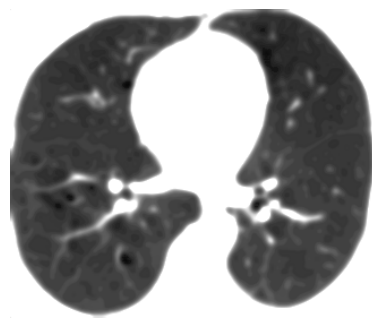

(n) \#317

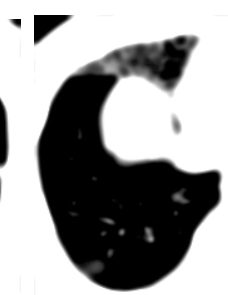

(c) \#35
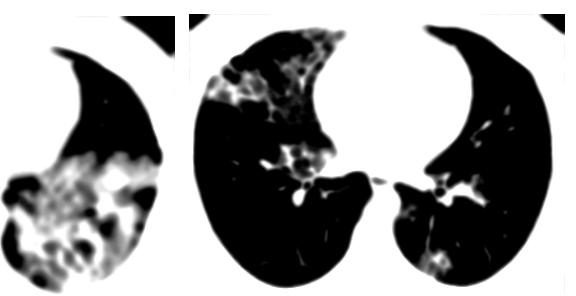

(d) \#55

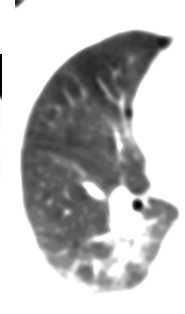

(g) \#434
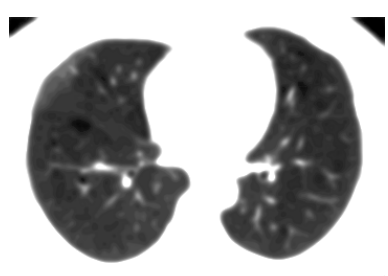

(k) \#121

(l) \#186

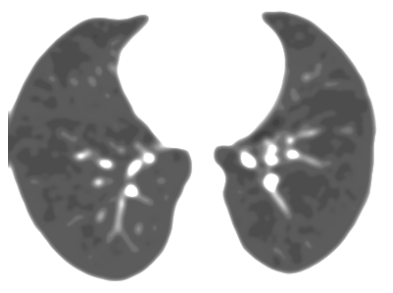

(h) \#1212
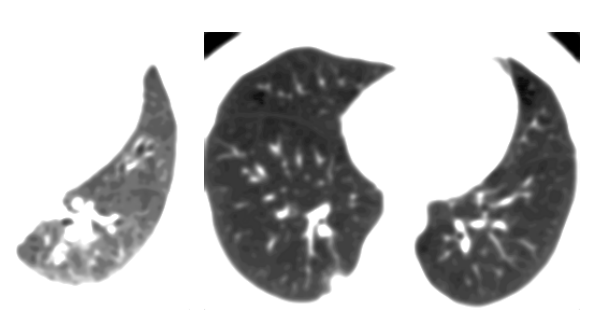

(l) \#186
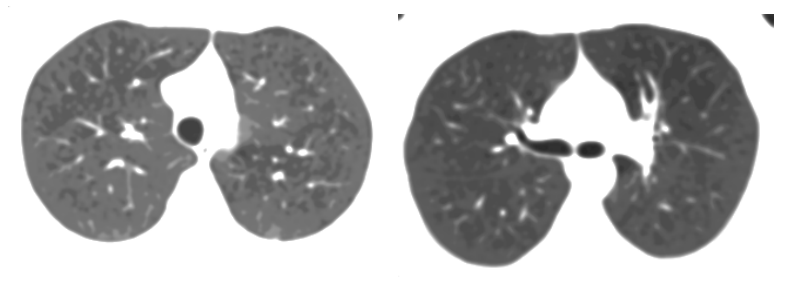

(o) \#842

(p) \#1217

Fig. 3: PQIS-Net segmented $(a-d)$ COVID-19, $(e-h) \mathrm{MP},(i-l) \mathrm{BP},(m-p) \mathrm{VP}$, and $(q-t)$ randomly selected lung CT slices [2]

[2] E. Soares, P. Angelov, S. Biaso, M. H. Froes, and D. K. Abe,"SARS-CoV-2 CT-scan dataset: A large dataset of real patients CT scans for SARS-CoV-2 identification," medRxiv, 2020, DOI: https://doi.org/10.1101/2020.04.24.20078584.

[3] T. Yan, "CCAP," IEEE Dataport, 2020, [Online]., Available: http://dx.doi.org/10.2127/ccgv-5329. Accessed: August 07, 2020.

[4] K. He, X. Zhang, S. Ren, and J. Sun, "Deep Residual Learning for Image Recognition," 2016 IEEE Conference on Computer Vision and Pattern Recognition (CVPR), 2016, DOI: 10.1109/CVPR.2016.90.

[5] J. Ma et al.,"Towards Efficient COVID-19 CT Annotation: A Benchmark for Lung and Infection Segmentation,”arXiv:2004.12537, 2020.

[6] Z. Han, B. Wei, Y. Hong, T. Li, J. Cong, X. Zhu, H. Wei, and W. Zhang, “Accurate Screening of COVID-19 Using Attention-Based Deep 3D Multiple Instance Learning,” IEEE Transactions on Medical Imaging, vol. 39, no. 8, August 2020, DOI: 10.1109/TMI.2020.2996256.

[7] J. Wang, Y. Bao, Y. Wen, H. Lu, H. Luo, Y. Xiang, X. Li, and C. Liu, "Prior-Attention Residual Learning for More Discriminative COVID-19 Screening in CT Images," IEEE Transactions on Medical Imaging, 2020, DOI: 10.1109/TMI.2020.2994908. 


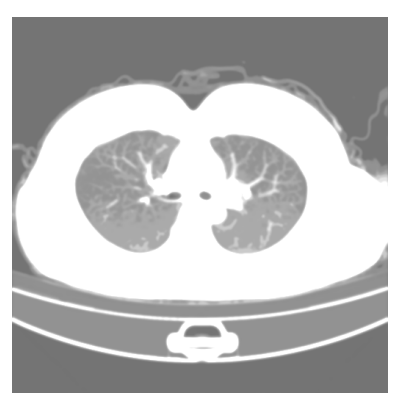

(a) \#4(1)

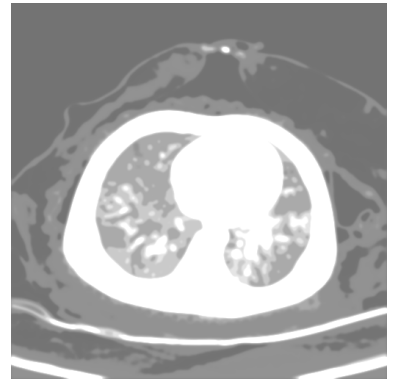

(e) \#5(1)

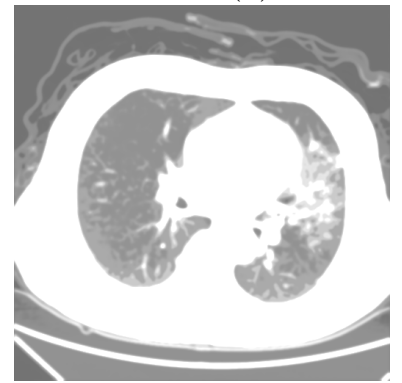

(i) \#5(1)

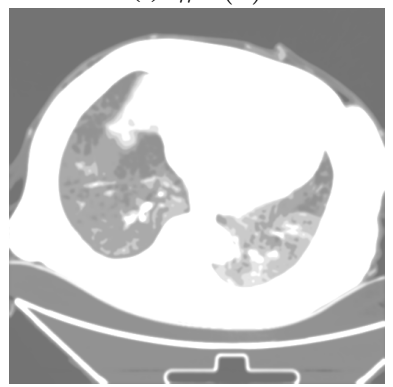

(m) \#41(1)

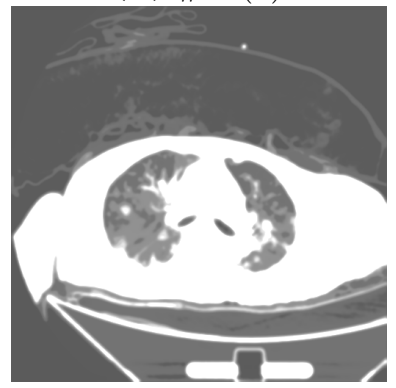

(q) \#24(47)

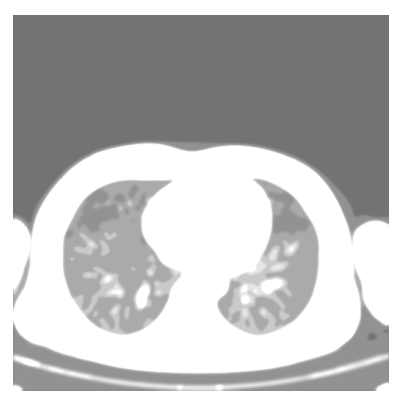

(b) \#6(220)

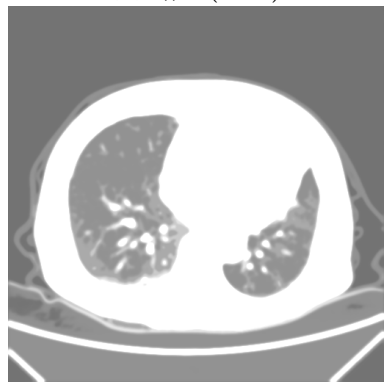

(f) $\# 3(7)$

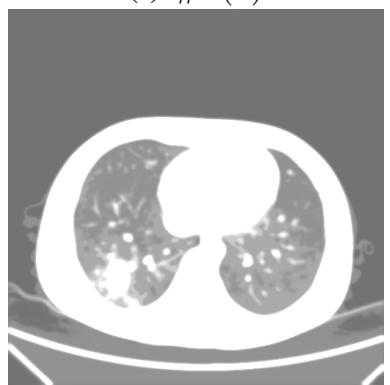

(j) \#12(10)

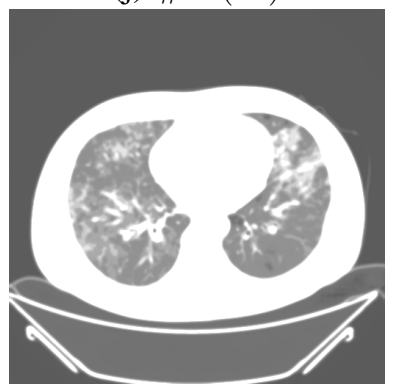

(n) \#58(20)

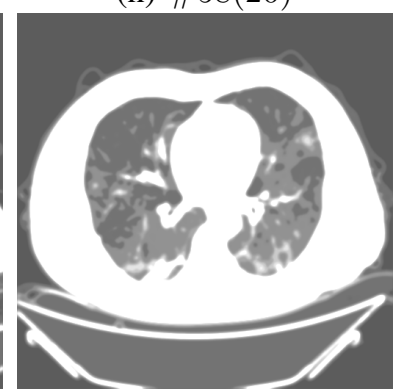

(r) \#25(147)

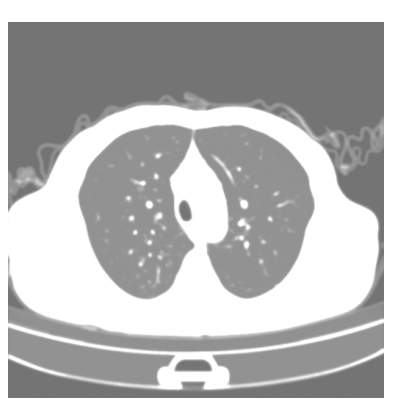

(c) \#18(240)

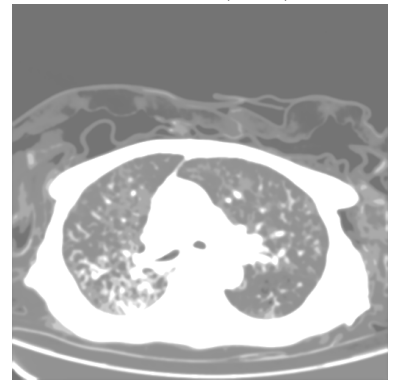

(g) \#40(76)

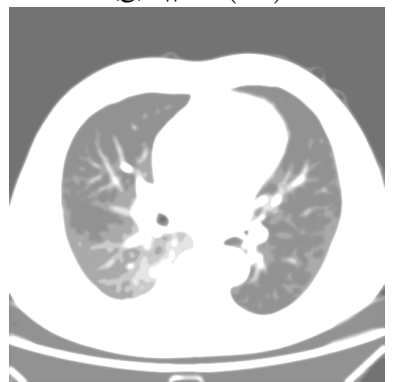

(k) \#46(60)

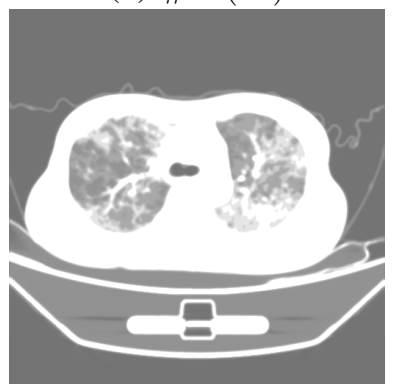

(o) \#22(45)

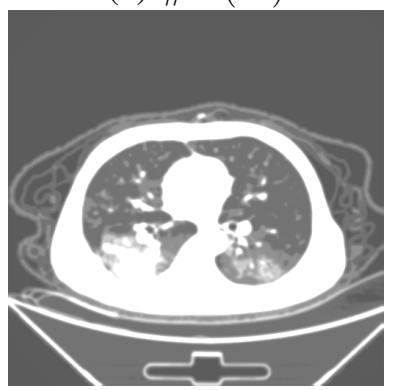

(s) \#24(47)

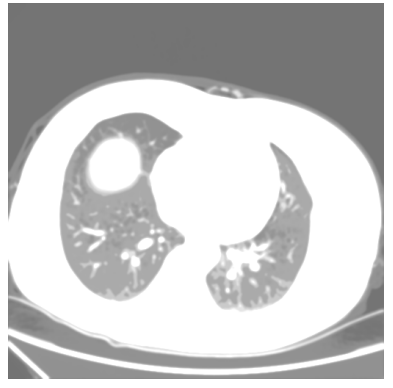

(d) \#24(245)

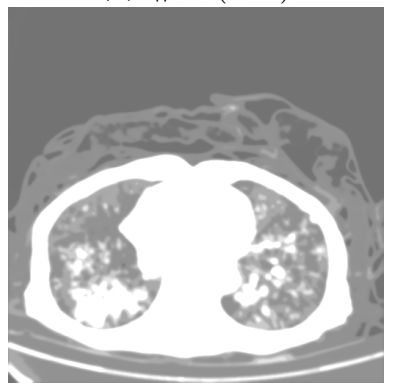

(h) \#90(76)

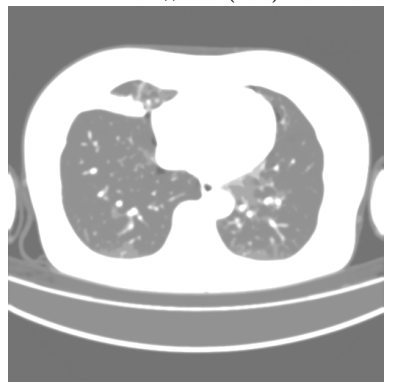

(1) \#51(80)

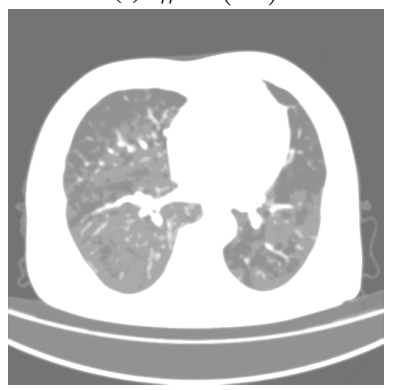

(p) \#81(55)

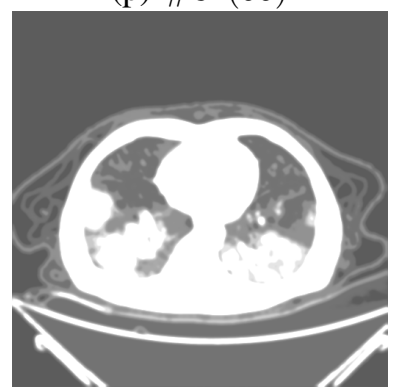

(t) \#24(47)

Fig. 4: PQIS-Net segmented $(a-d)$ Normal, $(e-h) \mathrm{MP},(i-l) \mathrm{BP},(m-p) \mathrm{VP}$, and $(q-t) \mathrm{CCAP}$ randomly selected lung CT slices (Patient\#) [3]. 


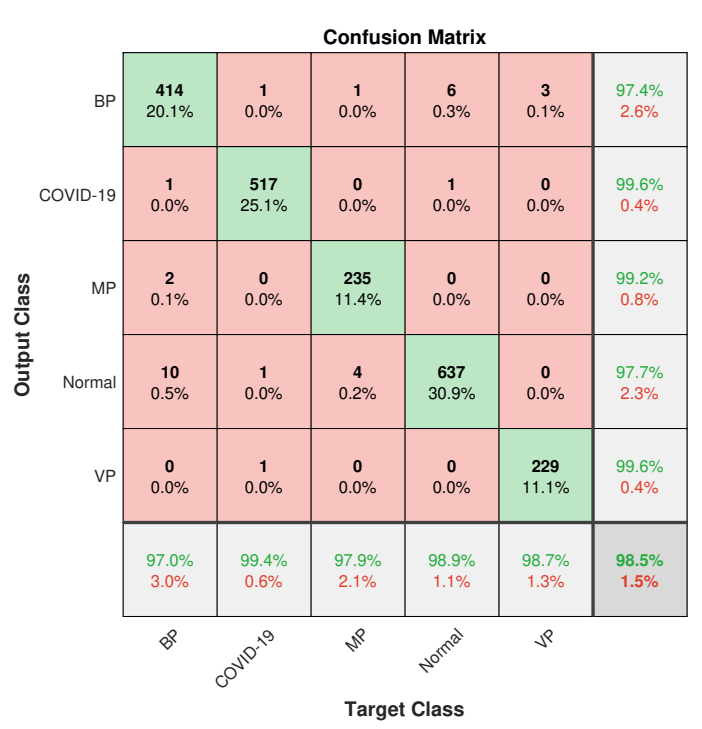

(a) ResNet50

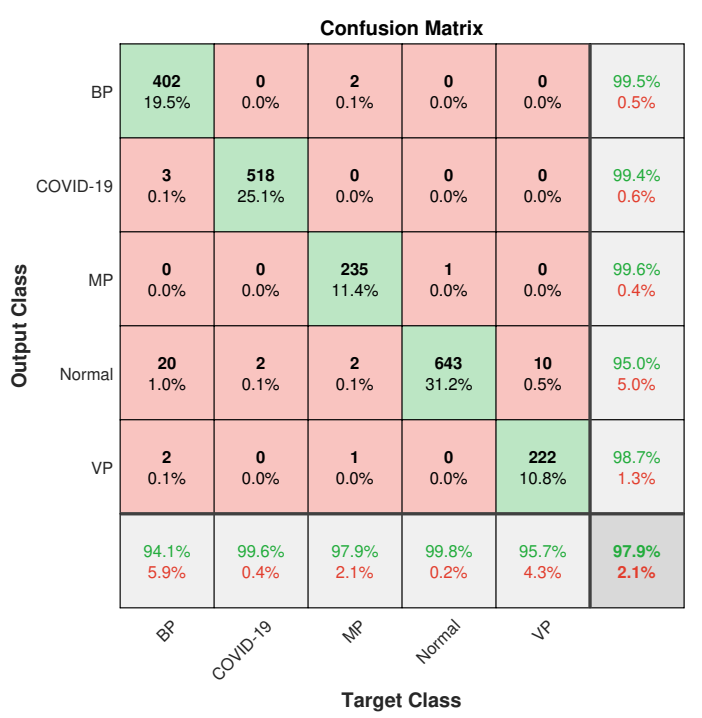

(c) $3 \mathrm{D}-\mathrm{UNet}$

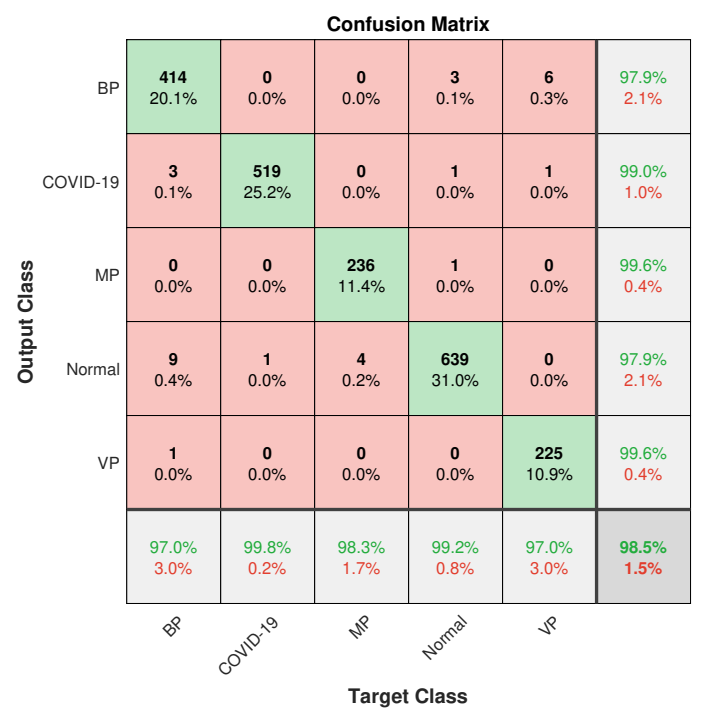

(b) Han et al.

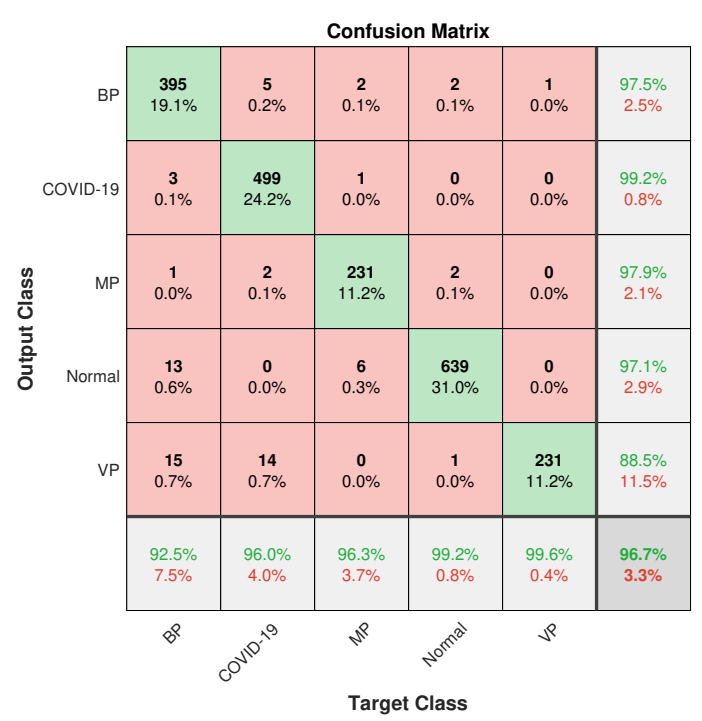

(d) Wang et al.

Fig. 5: Confusion matrices for the accuracy of prediction of Bacterial Pneumonia (BP), COVID-19, Mycoplasma Pneumonia (MP), Normal lung, and Viral Pneumonia (VP) using (a) ResNet50 [4], (b) 3D-UNet [5] (c) Han et al. [6], and (d) Wang et al. [7] on the data set [3]. 


\section{TABLE I: Description of the symbols used in the manuscript}

\begin{tabular}{|c|c|c|c|}
\hline Symbol & Description & Symbol & Description \\
\hline$\left|\phi_{i j}^{l}\right\rangle$ & $\begin{array}{l}\text { Each qubit is designated as } \phi_{i j}^{l} \text { at the } l^{t h} \text { layer } \\
\text { of PQIS-Net architecture }\end{array}$ & $x_{i}^{l}$ & $\begin{array}{l}\text { The classical input image pixel intensity }\left(x_{i}^{l}\right) \text { at } \\
\text { layer } l\end{array}$ \\
\hline$\omega^{l}$ & $\begin{array}{l}\text { The rotation angle for inter-connection strength } \\
\text { at layer } l\end{array}$ & $\gamma^{l}$ & The rotation angle for activation at layer $l$ \\
\hline$\mu_{i}^{l}$ & $\begin{array}{l}\text { The fuzzy graded input at the } i^{t h} \text { candidate } \\
\text { neuron at layer } l\end{array}$ & $\xi_{i}^{l}$ & $\begin{array}{l}\text { The fuzzy context sensitive activation in quantum } \\
\text { formalism at layer } l\end{array}$ \\
\hline$y_{i}^{l}$ & The output at the $i^{\text {th }}$ quantum neuron at layer $l$ & $\delta_{i}^{l-1}$ & The phase transformation parameters at layer $l$ \\
\hline$\tau$ & It is an imaginary number & $\mathcal{N}$ & $\begin{array}{l}\text { It corresponds spatially } \mathcal{N} \text {-connected second- } \\
\text { order neighborhood neuron }\end{array}$ \\
\hline$\sigma$ & $\begin{array}{l}\text { The Quantum Multi-level Sigmoidal (QMSig) } \\
\text { activation function }\end{array}$ & $\varphi_{j i}^{l}$ & The interconnection weight between at layer $l$ \\
\hline $\mathcal{N}_{s}$ & $\begin{array}{l}\text { The sum of the contribution of the } \mathcal{N} \text {-connected } \\
\text { neighborhood pixels }\end{array}$ & $\mathcal{S}_{\theta}$ & The outcome of two a class, $\theta$ \\
\hline$\nu$ & The steepness factor of the function QMSig & $L$ & $\begin{array}{l}\text { The number of gray levels in the segmented } \\
\text { image }\end{array}$ \\
\hline$\zeta$ & $\begin{array}{l}\text { The coherent network error cum loss function in } \\
\text { PQIS-Net }\end{array}$ & $\iota$ & $\begin{array}{l}\text { A particular epoch in PQIS-Net self-supervision } \\
\text { procedure }\end{array}$ \\
\hline $\mathcal{B}$ & $\begin{array}{l}\text { The batch size of the constituent PQIS-Net and } \\
\text { the semi-supervised model }\end{array}$ & $x_{p}$ & The center pixel of an image patch \\
\hline $\mathcal{R}_{p}$ & An image patch of size $s \times s$ & $s$ & Dimension of an patch \\
\hline $\mathcal{L}$ & The cross entropy loss function & $\Theta$ & $\begin{array}{l}\text { The hyper-parameters } \Theta \text { of the semi-supervised } \\
\text { neural network model }\end{array}$ \\
\hline$p$ & The number of FC layers & $\alpha_{i}$ & $\begin{array}{l}\text { The input to the FC layer of the semi-supervised } \\
\text { neural network model }\end{array}$ \\
\hline
\end{tabular}

